ERNEST DRLANDI LAWRENCE BERKELEY NATIONAL LABDRATIRY

\title{
Local Grid Refinement for Multi-Scale Geothermal Reservoir Simulation with TOUGH2
}

Julio García and Karsten Pruess

Earth Sciences Division

May 2000

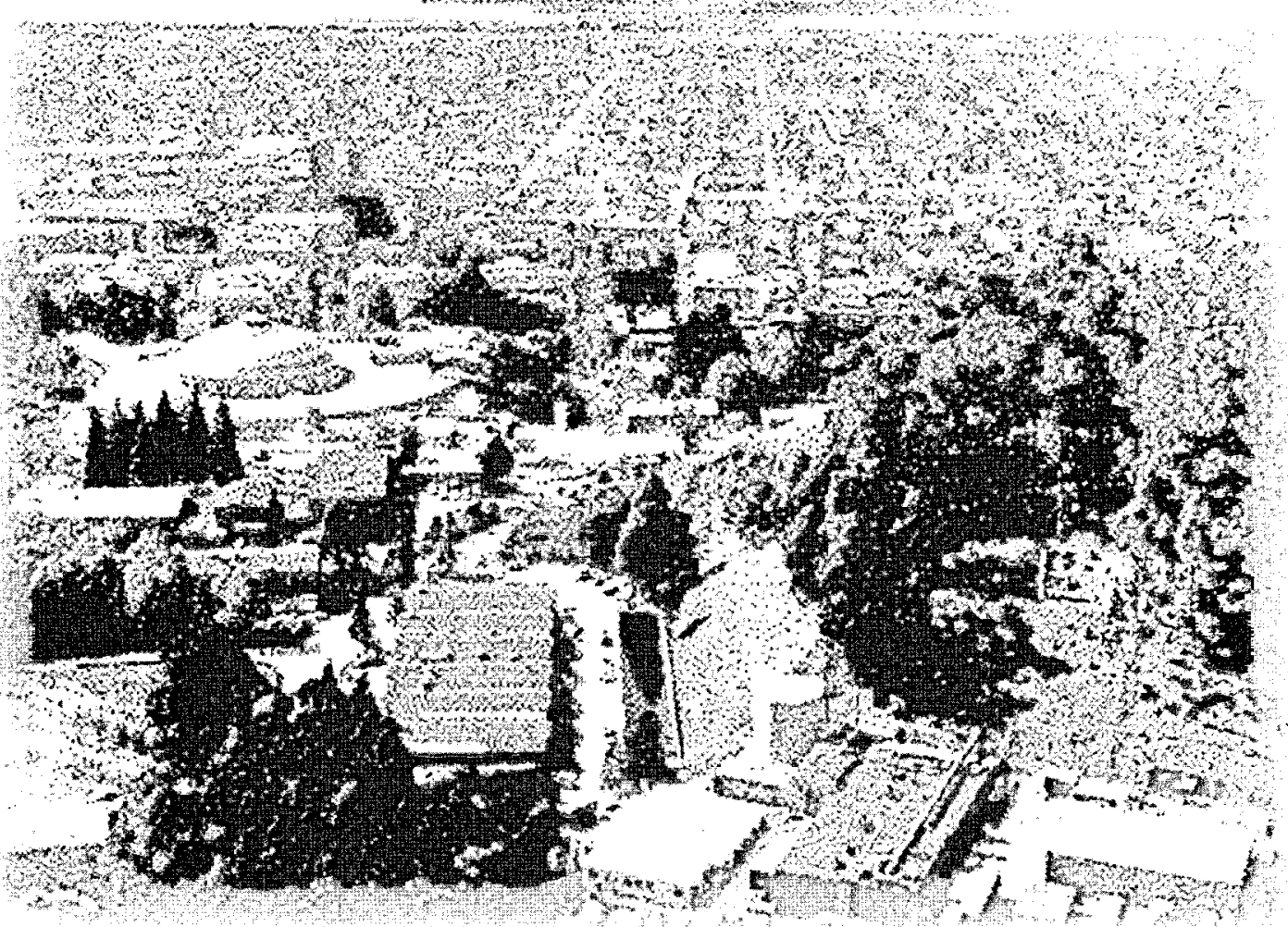




\section{DISCLAIMER}

This report was prepared as an account of work sponsored by an agency of the United States Government. Neither the United States Government nor any agency Thereof, nor any of their employees, makes any warranty, express or implied, or assumes any legal liability or responsibility for the accuracy, completeness, or usefulness of any information, apparatus, product, or process disclosed, or represents that its use would not infringe privately owned rights. Reference herein to any specific commercial product, process, or service by trade name, trademark, manufacturer, or otherwise does not necessarily constitute or imply its endorsement, recommendation, or favoring by the United States Government or any agency thereof. The views and opinions of authors expressed herein do not necessarily state or reflect those of the United States Government or any agency thereof. 


\section{DISCLAIMER}

Portions of this document may be illegible in electronic image products. Images are produced from the best available original document. 


\section{DISCLAIMER}

This document was prepared as an account of work sponsored by the United States Government. While this document is believed to contain correct information, neither the United States Government nor any agency thereof, nor The Regents of the University of California, nor any of their employees, makes any warranty, express or implied, or assumes any legal responsibility for the accuracy, completeness, or usefulness of any information, apparatus, product, or process disclosed, or represents that its use would not infringe privately owned rights. Reference herein to any specific commercial product, process, or service by its trade name, trademark, manufacturer, or otherwise, does not necessarily constitute or imply its endorsement, recommendation, or favoring by the United States Government or any agency thereof, or The Regents of the University of California. The views and opinions of authors expressed herein do not necessarily state or reflect those of the United States Government or any agency thereof, or The Regents of the University of California. 


\section{RECEIVED}

OCT 162000

OSTI

\section{LOCAL GRID REFINEMENT FOR MULTI-SCALE GEOTHERMAL RESERVOIR SIMULATION WITH TOUGH2}

Julio García and Karsten Pruess

Earth Sciences Division, Lawrence Berkeley National Laboratory

University of California, Berkeley, California 94720

May 2000

This work was supported by the Assistant Secretary for Energy Efficiency and Renewable Energy, Office of Geothermal and Wind Technologies, of the U.S. Department of Energy under contract No. DE-AC03-76SF00098 


\begin{abstract}
Thermodynamic conditions of geothermal reservoir fluids, such as pressures, temperatures, phase compositions, and concentrations of dissolved solids and non-condensible gases, may show strong spatial variability in the vicinity of production and injection wells. Large spatial variations also can occur near reservoir heterogeneities, such as faults and lithologic contacts. In numerical simulation of flow and transport, fine gridding is required to accurately represent steep changes in fluid conditions. This can necessitate prohibitively large numbers of grid blocks in conventional finite difference discretization, where finer gridding must be applied globally with reference to a global system of coordinates.
\end{abstract}

The objective of the work presented in this report was to develop a more economical alternative, in which fine gridding is applied only to regions in which large gradients in thermodynamic conditions need to be resolved. We have implemented a scheme for two-dimensional local grid refinement into the general-purpose geothermal reservoir simulator TOUGH2. We discuss the concept and applications to production from and injection into fractured two-phase reservoirs. The accuracy and economy of local grid refinement are demonstrated by comparison to simulations using conventional high resolution grids. 


\section{TABLE OF CONTENTS}

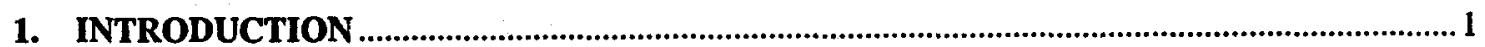

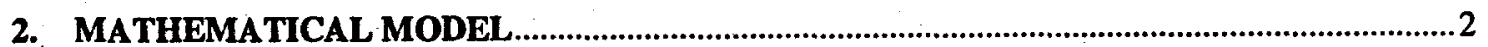

3. INTEGRAL FINITE DIFFERENCE DISCRETIZA TION …....................................................

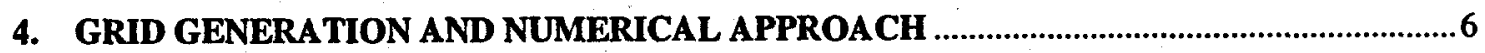

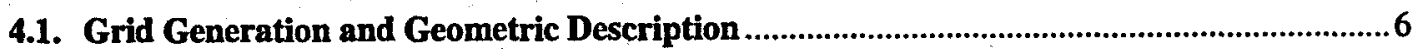

4.2. Numerical Approach and Approximation on Locally Refined Grids ................................. 14

4.2.1 Discretization in Locally Refined Grids.................................................................... 14

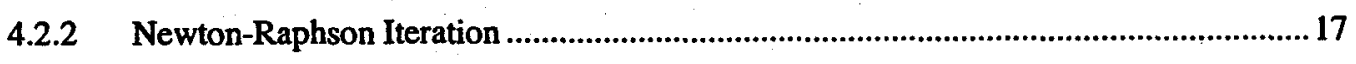

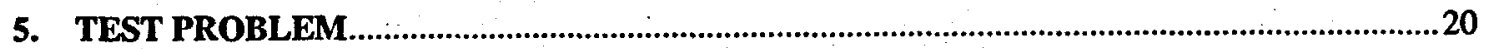

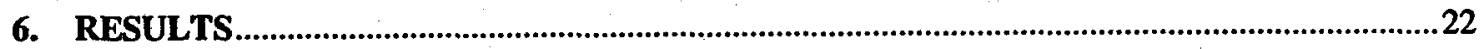

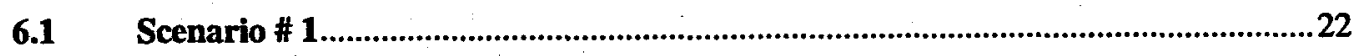

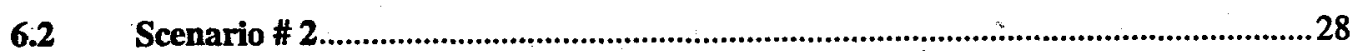

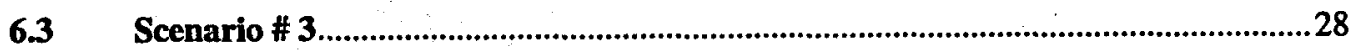

6.4 Economy of local grid refinement...........................................................................38

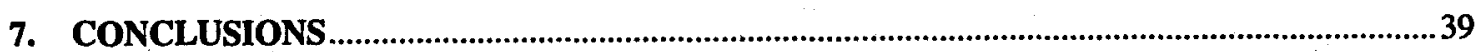

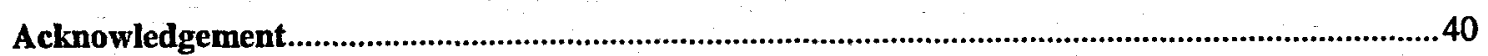



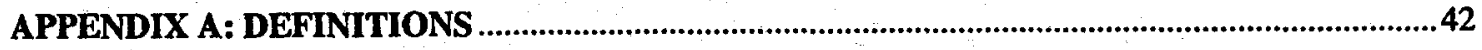

APPENDIX B: TOUGH2 INPUT FORMATS FOR GRID GENERATION

AND GEOMETRIC DESCRIPTION......................................................................43 


\section{LIST OF FIGURES}

Figure 1. Space discretization in the integral finite difference method ..............................................4

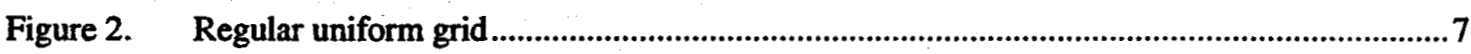

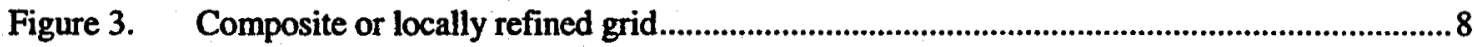

Figure 4. Reference elements in a locally refined grid .........................................................................11

Figure 5. Example of MESH file ............................................................................................................ 12

Figure 6. MESHMAKER input for generation of coarse and locally refined grids.............................14

Figure 7. Approximation of flow terms in locally refined grids.......................................................... 15

Figure 8. Linear Equation Setup ......................................................................................................19

Figure 9. Five-spot well pattern with local grid refinement for modeling $1 / 4$ symmetry domain .........21

Figure 10. Temperature and pressure comparison for fine, coarse and locally refined

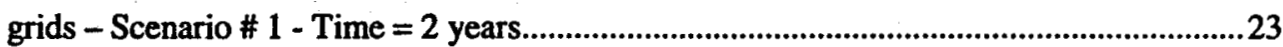

Figure 11. Relative error: Pressure - Scenario \# 1 - Time $=2$ years ...................................................2

Figure 12. Relative error: Temperature - Scenario \# 1 - Time $=2$ years ..........................................26

Figure 13. Saturation, temperature and pressure - Scenario \# 1 - Time $=8$ years...............................27

Figure 14. Saturation, pressure and temperature at a point near the injection well - Scenario \# 1 .......29

Figure 15. Saturation, pressure and temperature in the middle of the domain - Scenario \# 1...............30

Figure 16. Saturation, pressure and temperature at a point near the production well - Scenario \# 1 ....31

Figure 17. Saturation, temperature and pressure - Scenario \# 2 - Time = 1 year .................................32

Figure 18. Saturation, temperature and pressure - Scenario \# 2 - Time $=2$ years..................................33

Figure 19. Saturation, pressure and temperature at a point near the injection well - Scenario \# 2 ........34

Figure 20. Saturation, pressure and temperature in the middle of the domain - Scenario \# 2...............35

Figure 21. Saturation, pressure and temperature at a point near the production well -Scenario \# 2 .... 36

Figure 22. Saturation, temperature and pressure - Scenario \# 3 - Time = 1 year ...................................37

Figure 23. Illustration of MESH data for locally refined grid .......................................................45 


\section{LIST OF TABLES}

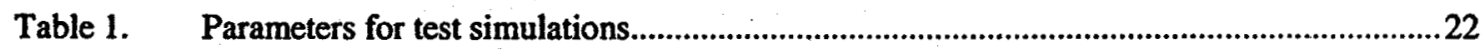

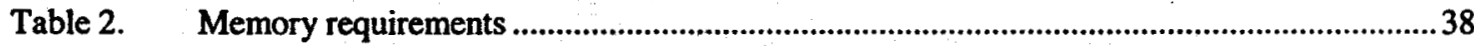

Table 3. Scenario \# 1 - CPU requirements (simulation time: 5 years) ..............................................38

Table 4. Scenario \# 2 - CPU requirements (simulation time: 2 years) ...........................................38

Table 5. Scenario \# 3 - CPU requirements (simulation time: 1 year) ....................................................39 


\subsection{INTRODUCTION}

Numerical simulation models are routinely used for approximate solution of the partial differential equations describing flow and transport in geothermal reservoirs. Many large scale physical processes have important local phenomena. For instance, pressure, temperature, and saturation can show locally large gradients near production and injection wells. The error associated with numerical simulations of such systems using coarse grids has been shown to be quite significant. For-example, saturation fronts that are often sharp can be smeared in numerical calculations (Peaceman, 1977). This phenomenon is usually described by the term numerical dispersion and it is due to truncation errors associated with the approximation of the differential equations using "coarse" grids. The finer the grid, the smaller the truncation error, therefore for some applications fine gridding is required to accurately represent steep changes in fluid conditions.

The objective of the work presented in this report was to develop a scheme for local refinement of 2-D Cartesian grids for the general-purpose geothermal reservoir simulator TOUGH2. Ultimately, the goal of the local grid refinement is to save computational resources within a given accuracy. For large-scale applications, the use of a uniform grid sufficiently fine to resolve the local phenomena gives rise to practical resource limitations, not only in terms of increased memory requirements but also because CPU times tend to increase quadratically or even cubically with mesh size (Chen et al., 1997).

Our main motivation for implementing local grid refinement within TOUGH2 is to improve the tradeoff between cost and accuracy associated with fine grids. There is a wide range of possible applications. In addition to accurately describing flow near wells, there is interest in simulating boiling and two-phase flow in fractures, migration of tracer fronts, and large spatial variations due to heterogeneities such as faults and lithologic contacts. The implementation of local grid refinement in the simulator TOUGH2 poses many challenges. Techniques have to be implemented to provide correct element and connection data and to store properly all reservoir parameters. Additionally, we need to consider a modified numerical approach that can handle the transition between coarsely gridded and locally refined regions. 
This report is organized in seven sections. The mathematical model and the integral finite difference discretization method are presented in sections 2.0 and 3.0 respectively. The material presented in these sections follows closely the presentation of Pruess (1987, 1991b), and is included here for completeness. Readers familiar with the TOUGH2 mathematical formulation are welcome to skip these sections. The core of the grid refinement approach is presented in section 4.0, where grid generation techniques and the numerical approach for uniform and locally refined grids are presented. Test problems and results are included in sections 5.0 and 6.0. Finally, section 7.0 provides some conclusions and suggestions for future developments.

\subsection{MATHEMATICAL MODEL}

The basic governing equation for mass and energy balances for multiphase nonisothermal flow is written in integral form for an arbitrary subdomain $V_{n}$ with surface area $\Gamma_{\mathrm{n}}$ as follows:

$$
\frac{d}{d t} \int_{V_{n}} M^{(\kappa)} d V=\int_{\Gamma_{n}} F^{(\kappa)} \cdot n d \Gamma+\int_{V_{n}} q^{(\kappa)} d V
$$

Here $M^{(\kappa)}$ denotes mass of component $\kappa(K=1, \ldots, N K)$ or energy $(K=N K+1)$ per unit volume. $F^{(x)}$ is mass (or heat) flux entering $V_{n}$ at the surface $\Gamma_{n}, n$ is a unit normal vector pointing into $V_{n}$, and $q^{(k)}$ represents sinks or sources. The general form of the mass accumulation term is:

$$
M^{(\kappa)}=\phi \sum_{\beta=1}^{N P H} S_{\beta} \rho_{\beta} X_{\beta}^{(\kappa)}
$$

Here NPH is the total number of phases, $S_{\beta}$ is the saturation (volume fraction) of phase $\beta$, $\rho_{\beta}$ is the density of phase $\beta$, and $X_{\beta}{ }^{(\kappa)}$ is the mass fraction of component $\kappa$ present in phase $\beta$, and $\phi$ is the porosity. Similarly, the heat accumulation term in a multi-phase system is (including fluid and rock contributions) 


$$
M^{(N K+1)}=\phi \sum_{\beta=1}^{N P H} S_{\beta} \rho_{\beta} u_{\beta}+(1-\phi) \rho_{R} C_{R} T
$$

where $\rho_{R}$ and $C_{R}$ are, respectively, density and specific heat of the rock grains, $T$ is the temperature, and $u_{\beta}$ is the specific internal energy of phase $\beta$.

The mass flux is the sum of component $\kappa$ fluxes in all phases. Individual phases mass fluxes are given by Darcy's law extended to multiphase flow:

$$
F^{(\kappa)}=\sum_{\beta=1}^{N P H} X_{\beta}^{(\kappa)} F_{\beta}=-k \sum_{\beta=1}^{N P H} X_{\beta}^{(\kappa)} \frac{k_{r \beta}}{\mu_{\beta}} \rho_{\beta}\left(\nabla P_{\beta}-\rho_{\beta} g\right)
$$

Here $k$ denotes the absolute permeability tensor, $k_{\alpha \beta}$ is relative permeability of phase $\beta, \mu_{\beta}$ is viscosity, $P_{\beta}$ is the pressure in phase $\beta$, which is the sum of the pressure $P$ of a reference phase and the capillary pressure of phase $\beta$ relative to the reference phase, and $\mathbf{g}$ is the vector of gravitational acceleration. Heat flux in general contains conductive and convective components, i.e.,

$$
F^{(\mathrm{NK}+1)}=-\mathrm{K} \nabla \mathrm{T}+\sum_{\beta=1}^{\mathrm{NPH}} \mathrm{h}_{\beta} \mathrm{F}_{\beta}
$$

where $K$ is the thermal conductivity of the rock-fluid mixture, and $h_{\beta}$ is the specific enthalpy of phase $\beta$.

\subsection{INTEGRAL FINITE DIFFERENCE DISCRETIZATION}

The mass and energy balance equations in (2.1) are discretized in space using the integral finite difference method (Narasimham and Witherspoon, 1976). Introducing appropriate volume and surface area averages, a set of first-order ordinary differential equations in time is obtained.

$$
\frac{d M_{n}^{(\kappa)}}{d t}=\frac{1}{V_{n}} \sum_{m} A_{n m} F_{n m}^{(\kappa)}+q_{n}^{(\kappa)}
$$


Here $M_{n}$ is the average value of $M$ over $V_{n}, A_{n m}$ is the interface area between grid blocks $n$ and $m$, and $F_{n m}$ is the average of flux $F$ over $A_{n m}$ (Figure 1). The summation is over all grids blocks $m$ that are connected to $n . F_{n m}^{(\kappa)}$ is the discretized flux of component $k$ between grid blocks $\mathrm{n}$ and $\mathrm{m}$. Moreover $\mathrm{F}_{\mathrm{nm}}^{(\mathrm{k})}$ is the summation of fluxes over all phases:

$$
F_{\mathrm{nm}}^{(\boldsymbol{k})}=\sum_{\beta=1}^{\mathrm{NPH}} \mathrm{F}_{\beta, \mathrm{mm}}^{\mathrm{k}}
$$

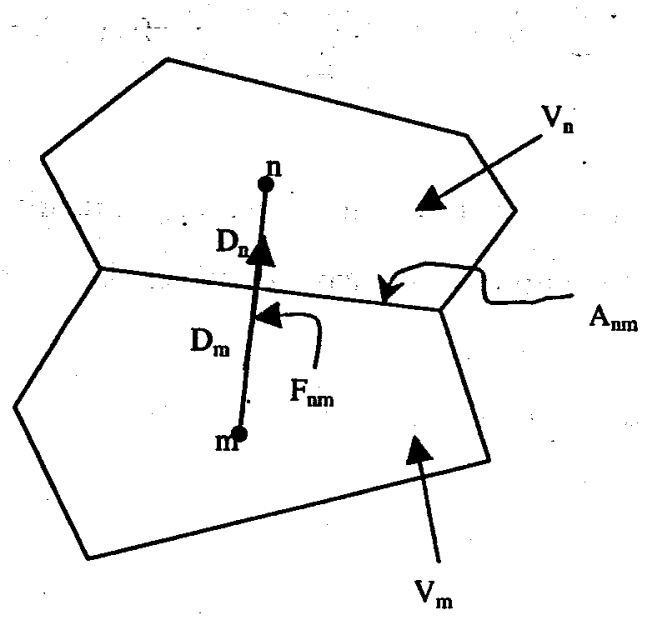

Figure 1

\section{Space discretization in the integral finite difference method}

Individual phase fluxes are expressed in terms of averages over parameters for elements $\mathrm{V}_{\mathrm{n}}$ and $\mathrm{V}_{\mathrm{m}}$. A finite difference approximation for the basic Darcy flux term (Equation 2.4 ) is given by:

$$
F_{\beta, n m}=-k_{n m}\left[\frac{k_{r p} \rho_{\beta}}{\mu_{\beta}}\right]_{n m}\left[\frac{P_{\beta, n}-P_{\beta, m}}{D_{n m}}-\rho_{\beta, n m} g_{n m}\right]
$$


where the subscripts $(n m)$ indicate that the quantities $k_{n m},\left[\frac{k_{\beta \beta}}{\mu_{\beta}}\right]_{n m}, \rho_{\beta, n m}$ (permeability, mobility and density, respectively) are evaluated at the interface between elements $m$ and n using a suitable averaging technique (interpolation, harmonic weighting, upstream weighting). Finally, $D_{n m}$ is the distance between the nodal points $\mathbf{n}$ and $\mathbf{m}$, and $g_{n m}$ is the component of gravitational acceleration in the direction of $m$ to $n$.

Time is discretized fully implicitly as a first order finite difference, and the flux and sink/source terms on the right-hand side of equation (3.1) are evaluated at the new time level, $\mathrm{t}^{\mathrm{k}+1}=\mathrm{t}^{\mathrm{k}}+\Delta \mathrm{t}$. This ensures the numerical stability needed for an efficient calculation of multi-phase flow. The time-discretized version of equation 3.1 can be written in terms of mass component and energy residuals as the following set of coupled non-linear, algebraic equations.

$$
R_{n}^{(k) k+1}=M_{n}^{(x) k+1}-M_{n}^{(k) k}-\frac{\Delta t}{V_{n}}\left\{\sum_{m} A_{n m} F_{n m}^{(k) k+1}+V_{n} q_{n}^{(k) k+1}\right\}=0
$$

For each volume element (grid block) $V_{n}$ there are NEQ equations, so that in a flow domain with NEL grid blocks, (3.4) represents a total of NEL*NEQ coupled non-linear equations. The unknowns are the NEL*NEQ independent primary variables $\left(x_{i} ; i=1, \ldots\right.$, NEL*NEQ) which are obtained by Newton/Raphson iteration.

Newton/Raphson iteration is implemented in TOUGH2 as follows. A Taylor series expansion of the residuals $R_{n}{ }^{(k) k+1}$ in equation (3.4) about an assumed solution $x_{p+1}$ (iteration step $\mathrm{p}+1$ ) is performed:

$$
R_{n}^{(k) k+1}\left(x_{i, p+1}\right)=R_{i}^{(k) k+1}\left(x_{i, p}\right)+\left.\sum_{i} \frac{\partial R_{n}^{(k) k+1}}{\partial x_{i}}\right|_{p}\left(x_{i, p+1}-x_{i, p}\right)+\ldots . .=0
$$

Retaining only first order terms, we obtain a set of NEL*NEQ linear equations for the increments $\left(\mathbf{x}_{\mathrm{i}, \mathrm{p}+1}-\mathbf{x}_{\mathrm{i}, \mathrm{p}}\right)$ : 


$$
-\left.\sum_{i} \frac{\partial R_{n}^{(k) k+1}}{\partial x_{i}}\right|_{p}\left(x_{i, p+1}-x_{i, p}\right)=R_{n}^{(k) k+1}\left(x_{i, p}\right)
$$

All terms $\partial R_{n} / \partial x_{i}$ represents entries of the Jacobian matrix that are evaluated by numerical differentiation. The system of simultaneous equations is solved with the aid of a linear equation solver. Iteration is continued until all residuals $\mathbf{R}_{\mathbf{n}}^{(\mathrm{(x)k+1}}$ are reduced below a preset convergence tolerance (see Pruess, 1987).

\subsection{GRID GENERATION AND NUMERICAL APPROACH}

\subsection{Grid Generation and Geometric Description}

Because there is no reference to a global system of coordinates or to dimensionality of a particular problem, the geometric description implemented within TOUGH2 (list of element volumes $V_{n}$, interface areas $A_{n m}$, and nodal distances $D_{n m}$ ) permits one-, two-, and three-dimensional regular or irregular grid systems. However, the integral finite difference grid discretization requires that the line connecting nodal points for adjacent volume elements coincide with the principal axis of permeability and that the interface area is perpendicular to this line. The existence of thermodynamic equilibrium in grid blocks at (almost) all times is also required.

For grid systems of regular blocks referred to a fixed global coordinate system, the Integral Finite Difference method reduces to conventional finite differences. Although limiting the geometric description, the use of cartesian grids involving global coordinates $(\mathrm{x}, \mathrm{y}, \mathrm{z})$ has several advantages. For example, the resulting sparse Jacobian matrix has a regular banded structure (Moridis and Pruess, 1998; Aziz and Settari, 1979), which simplifies and reduces the cost of solving the set of numerical finite difference equations. Cartesian grids are commonly used not only because numerical solutions might be less expensive but also because they are simpler to generate and handle. It is within the framework of cartesian grids that the proposed local refinement of grids for TOUGH2 is developed. 
It is appropriate, at this point, to introduce the grid generation technique implemented within TOUGH2. One, two, or three-dimensional cartesian $X-Y-Z$ grids are created invoking the sub-module MESHMAKER and keywords ' $X Y Z$ '. Subroutine GXYZ is called and a file MESH is created with volume element (ELEME) and connection (CONNE) geometric descriptions. The reader is referred to the TOUGH2 user's guides for additional details (Pruess 1987; 1991b; Pruess et al., 1999). The grid represented in Figure 2 illustrates how meshes are generated. For two-dimensional grids ordering of elements is performed first along the $y$ direction ( $j$ increment) and then along the $x$ direction (i increment). The numbering and ordering of connections proceed in similar fashion. For the grid in Figure 2, $\mathrm{I}=4$ and $\mathrm{J}=3$, we have 12 elements or grid blocks and 17 connections or interfaces between them.



Figure 2

Regular Uniform Grid

Now, let us turn our attention to local grid refinement. The ultimate purpose is to increase the number of blocks in a particular area of interest such as around injection/production wells and lithologic contacts. For illustration purposes, let us assume that we want to refine the grid of Figure 2 in the vicinity of a hypothetical well located in the center of element 5 . The new locally refined grid is created by subdividing volume element \# 5 
into smaller blocks. In the example shown in Figure 3, element \# 5 is partitioned into $3 \mathrm{x}$ $3=9$ blocks.

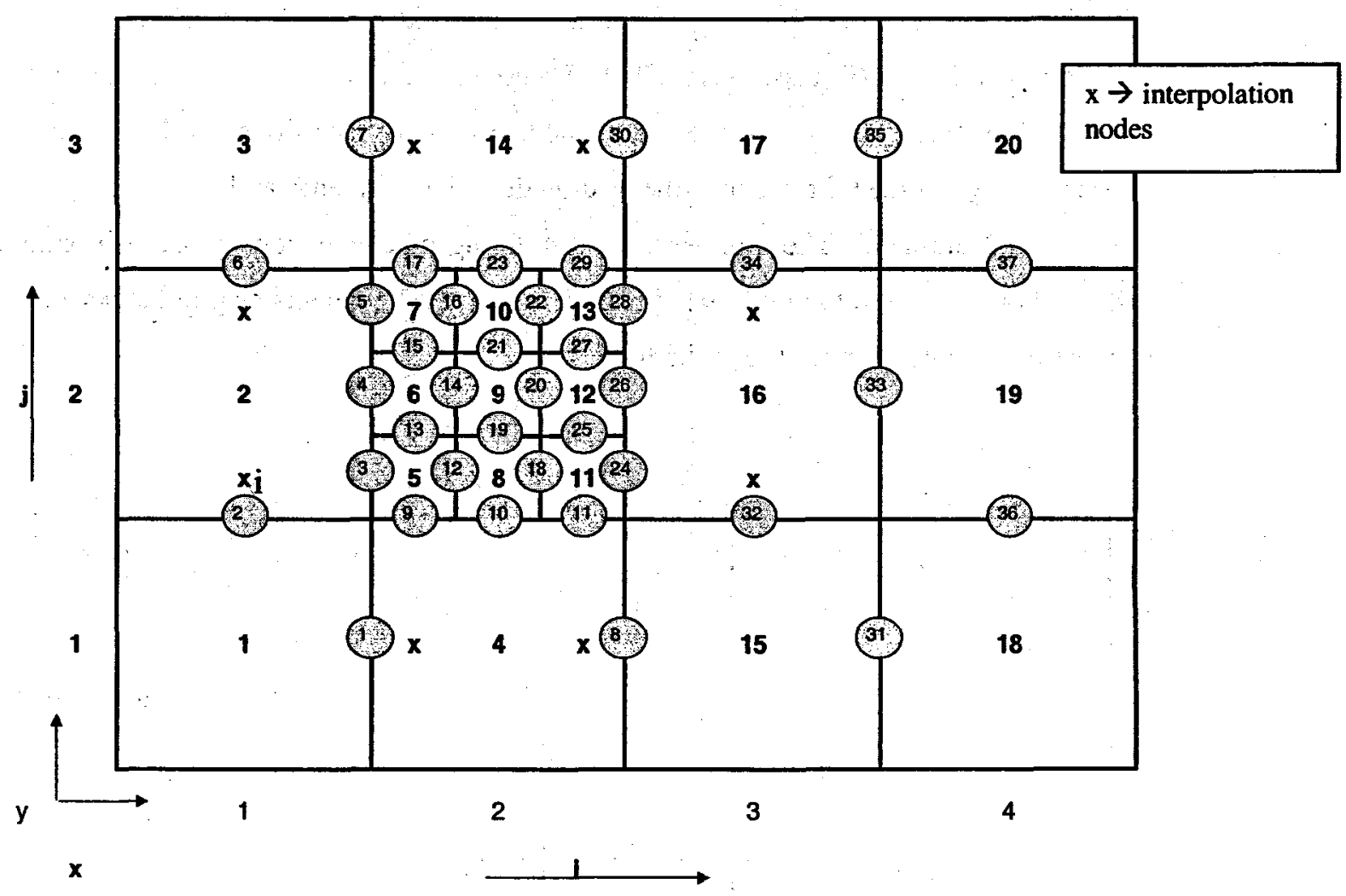

Figure 3

\section{Composite or Locally Refined Grid}

After the local refinement is performed, several observations can be made. First and most obviously, we notice an increase in the number of elements and connections. In order to accommodate new elements and connections some of the non-refined or regular grid blocks have now "additional" interfaces (grid blocks 2,4,6, and 8 in Figure 2; 2, 4, 14 and 16 in Figure 3). In fact some connections that were previously regular connections (interface between two regular blocks), are now divided into three to form what we will call Composite Connections (See list of definitions in Appendix A). The second and more relevant change entails the presence of adjacent volume elements (grid blocks) whose nodal lines (connection between nodal points) do not run perpendicular to their common interface. 
In TOUGH2 the pressure gradient that drives the fluid flow across the interface between grid blocks 1 and 2 is approximated as

$$
(\nabla P)_{12} \cong\left(P_{1}-P_{2}\right) / D_{12}
$$

where $D_{12}$ is the nodal distance between points 1 and 2 . The integral finite difference method used in TOUGH2 (see Section 3.0) requires that the nodal line connecting nodal points 1 and 2 be perpendicular to the interface between the respective grid blocks, in order that Equation (4.1) may represent the normal component of the pressure gradient with respect to this interface. In the locally refined grid shown in Figure 3, a problem now arises in the computation of the pressure gradient at the interfaces between the smaller and larger blocks. In general, nodal lines running from smaller grid blocks perpendicular to their interfaces with blocks $2,4,14$, and 16 will not intersect a nodal point on the other side. This situation is remedied by introducing interpolation nodes at which the required information can be obtained by spatial interpolation from neighboring grid nodes. For example, Figure 3 shows an interpolation node labeled "i", which is located at the intersection between the nodal line originating from grid block \# 5 , and the nodal line between 1 and 2 . Fluid pressure at $i$ is obtained by linear interpolation between 1 and 2,

$$
P_{1}=P_{2}+\left(P_{1}-P_{2}\right) D_{i 2} / D_{12}
$$

where $D_{i 2}$ is the distance between points $i$ and 2 . The normal component of pressure gradient at the interface between blocks 2 and 5 is then written as

$$
(\nabla P)_{25} \equiv\left(P_{i}-P_{5}\right) / D_{i s}
$$

where $D_{i s}$ is the distance between nodes $i$ and 5 . The same interpolation scheme is used for other parameters that are needed to calculate mass and heat fluxes at the interface between 2 and 5 , including temperature, relative permeability, viscosity, density, enthalpy, capillary pressure, and mass fractions of solutes or non-condensible gases. 
It is clear that the geometric description currently implemented within TOUGH2 does not provide all the information required to fully characterize locally refined grids, and that a new extended data structure for connection information is needed. Additionally, an improved numerical scheme is needed for grid blocks belonging to different refinement zones to consistently calculate fluxes (Equation 3.3), residuals (Equation 3.4) and Jacobian terms (Equation 3.6). The new geometric description must be capable of differentiating between regular and composite connections, and identifying reference elements to approximate fluid and rock properties at interpolation nodes.

It is appropriate to remind the reader that the data handling in TOUGH2 is accomplished by means of disk files. All the modifications to the geometric description are included in the data block CONNE (Connection data) in the disk file MESH. Connection data information includes pairs of elements, interface area, nodal distances from the interface, and orientation of the nodal line relative to gravity, which is assumed parallel to the $Z$ coordinate. In locally refined grids, modifications to connection data are performed only for composite connections. Interface area is set to a negative value allowing the new module to distinguish this type of connections from regular ones. The following additional information is also appended at the end of each composite connection data line:

- Reference element: In order to obtain fluid properties at the interpolation nodes, the current implementation considers an interpolation between the parameter values at the actual center of the grid block and the values at the reference element node. In the grid shown in Figure 4, to calculate fluxes in and out of $P$, usually we need data from grid blocks $N, E, S, W$; if $P$ is refined, we also need information from NE, SE, SW and NW blocks (reference elements).

- Location Index: Knowledge of the location of the interpolation node within the special element is also required. The current implementation allows the refinement of a coarse element into any odd number of blocks. Thus, it is necessary to specify the location of interpolation nodes relative to the actual node. The Location Index data variable is introduced for that purpose. It allows not only to specify the relative location of the interpolation node between the actual and the reference element but 
also to compute the interpolation coefficients required for estimating fluid properties at this location.

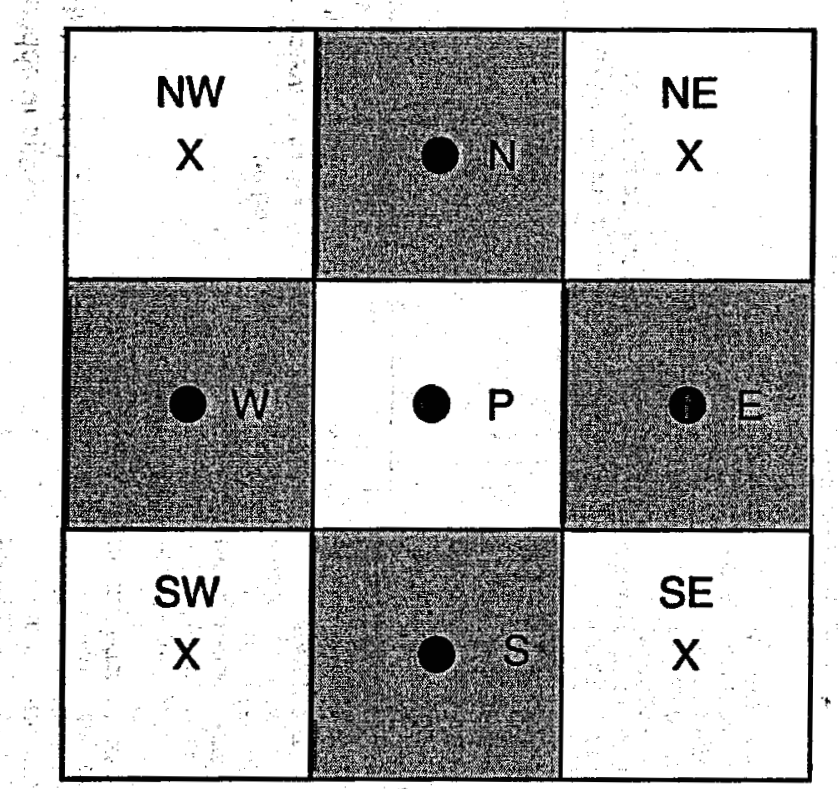

Figure 4

\section{Reference Elements in a Locally Refined Grid}

- Coefficient of Refinement: Finally, the coefficient of refinement indicates how many composite connections are present. This value is also used to compute interpolation coefficients.

Figure 5 shows the corresponding MESH file for the grid presented in Figure 4 with a coefficient of refinement $\mathrm{CR}=3$. Note that composite connections have negative interface area and the presence of the three additional information data entries explained above. Enhancements in the code allow recognizing the new (or modified) input connection data, yet maintaining compatibility with standard TOUGH2 data blocks.

The definition of the flow geometry can be further simplified by using the program module MESHMAKER. Modifications to subroutine GXYZ allow the user to generate locally refined grids by specifying the boundaries of the refined region (Coordinates XR1, XR2, YR1, YR2), and the coefficient of refinement. Note that if a coefficient of refinement equal to $n$ is applied to a coarse grid block, this will be divided into $n^{2}$ fine grid blocks ( $n$ by $n$ ). 


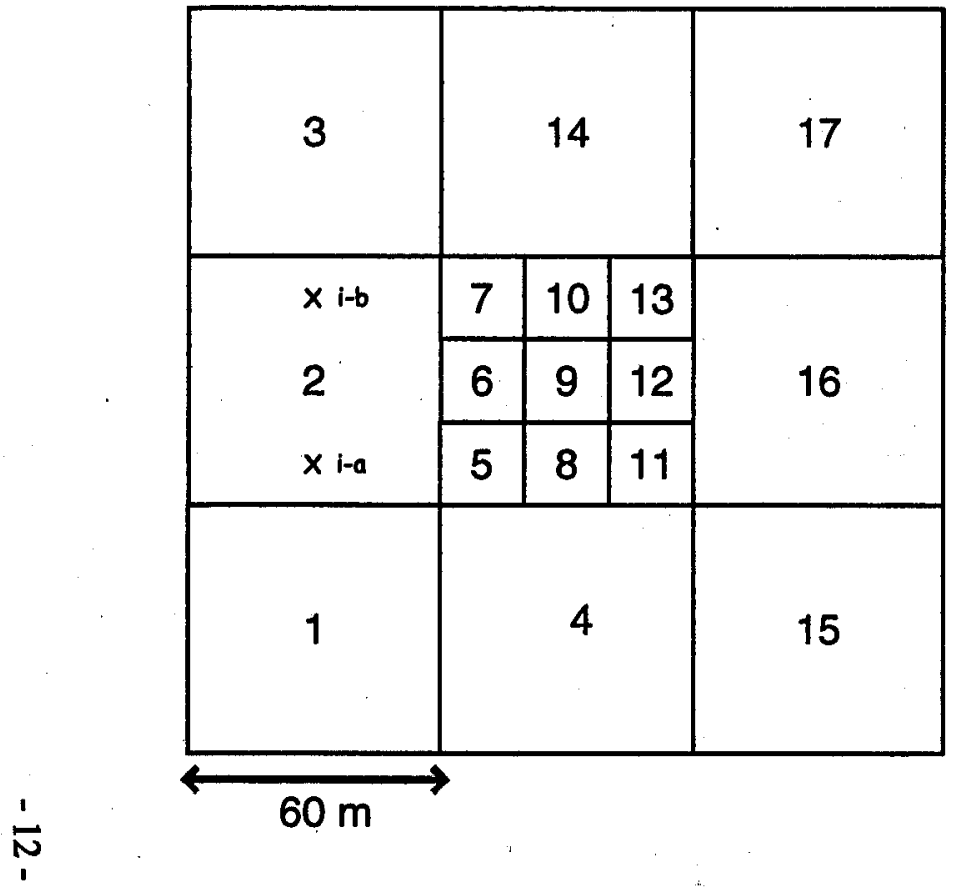

Figure 5

Example of Mesh File (thickness is $15 \mathrm{~m}$ )

Comments: Connections such as 2-6, 4-8, etc. that are identified as "composite" with location index 0 can be considered "normal" or "regular" connections, as they require no interpolation and will in all respects be treated as non-refined connections.
$10.54008+050.72008+04$

$10.54008+050.72008+04$

$10.54008+5050.72008+04$

$10.54008+050.72008+04$

$10.60008+040.80008+63$
$10.60008+040.80008+63$

$10.60008+040.80008+03$

$10.60008+040.60008+03$

10. $60008+040.80008+03$

10. $6000 \mathrm{~B}+040.8000 \mathrm{~B}+03$

10. $6000 \mathrm{z}+040.80008+03$

10. $60008+040.80008+03$

$10.60008+040.80008+03$

$10.54008+050.72008+04$

$10.51002+050.72008+04$

$10.510035050 .72008+01$
$0.30008+020.30008+02-.75008+02$ $0.30008020 .15003+03-75008+01$ $0.70005+020.70005+02-.75008+01$ $0.70008+220.90008+02-.75008+01$ $0.70008+020.11008+03-.75008+01$ $0.90008+020.70008+02-.75008+01$ $0.90008+020.90008+02-.75008+01$ $0.90008+020.11008+03-.75008+01$ $0.11008+030.70008+02-.75008+02$ $0.11008+030.90008+02-.15008+01$ $0.11008+030.11008+03-.15008+01$ $0.90008+020.1500 \mathrm{~B}+03-.7500 \mathrm{Z}+02$ $0.15008+030.3000 \mathrm{~B}+02-.7500 \mathrm{~B}+01$ $0.1500 \mathrm{E}+030.9000 \mathrm{~B}+02-.7500 \mathrm{~B}+02$
$0.15008+030.2500 \mathrm{z}+03-.7500 \mathrm{z}+01$ $0.90005+020.30003+02-75005+01$

Element 2 has 3 composite connections. They are identified by a negative interface area. To calculate fluxes across the composite connections two interpolations nodes are required ( $i-a$ and $i-b$ ). Reference element for $i-a$ is 1 . Reference element for $i-b$ is 3. The Location Index ( $L I=1)$ for both interpolation nodes is 1 since there is only one interpolation node between the actual and reference element. The Coefficient of Refinement in this case is 3 (CR=3)

Coefficients of interpolation are $1 / 3$ (LI/CR) and $1-1 / 3$

$10.30008+020.30008+220.90008+03$ $20.30008+020.30008+220.90008+030.00008+00$ $10.30005020 .1000902-3000503$ $10.30008+020.10008+02-30008+03$ 20.30008+020:30008+020.90008+030.00008+ +00 $10.30008+020.30008+220.90008+03$ $10.30008+020.30005+020: 90008+63$

$20.30008+020.10008+12-.30008+030.00008+00$ $20.30003+020.10008+02-30008+030.00005+00$ $20.30008+020.1000 B+02-30008+030.00008+00$ $10.10008+020.10008+020.30008+03$

$20.10008+020.10008+020.3000 \mathrm{~B}+030.00008+00$ 10.10008+ $+220.10008+020.30008+03$ $20.1000 z+020.10008+020.30003+030.00008+00$ $10.10008+020.10008+020.30008+03$ $20.10008+020.30008+02-30008+030.0006$ $10.1002+20.10003+020.30003+03$

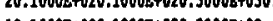
$10.1002+02.10035020 .3000403$

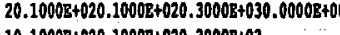

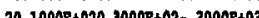

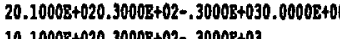
$20.10005+020.00008+020.30008+030.00$

$10.10003+020.30008+02-.30008+03$ $20.10008+020.10008+020.30003+030.00008+100$ $10.10003+020.30008+02-3000 B+03$ $\begin{array}{lllll}10.10008+020.30008+02-.30008+03 & -17 & 1 & 3 \\ 20.10008+020.30008+202-3008+030.00008+00 & -17 & 1 & 3\end{array}$ 10.30008+020.30008+020.90008+03 $20.30008+020.30008+020.9000 B+030.00008+00$ $-14: 3$ $\begin{array}{llll}-15 & 1 & 3\end{array}$ $-1603$

Note: See additional details in appendix B

The reference element is in this case set to a negative value in order to identify connections where the first element is inside a locally refined region.

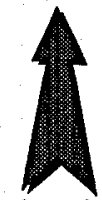

Additional Information in Connnection Data 
The input for MESHMAKER has a modular structure and a variable number of records; it begins with keyword 'MESHM' and ends with a blank record. A description of available MESHMAKER sub-modules is provided in Pruess (1991b) and Pruess et al. (1999). Keyword XYZ invokes the generation of a cartesian (rectilinear) mesh; records XYZ.1, XYZ.2 and XYZ.3 provide the geometric description. Two additional records are required to create locally refined grids.

\section{Record XYZ.4}

Format(A2,3X,I5)

NTYPE, CRLRZ

NTYPE Set equal to LR for specifying Local Refinement Zone

CRLRZ Coefficient of Refinement desired

\section{Record XYZ.5}

Format (4E10.4)

XR1,XR2,YR1,YR2

XR1 Left $x$ coordinate for local refinement zone.

XR2 Right $x$ coordinate for local refinement zone.

YR1 Lower y coordinate for local refinement zone.

YR2 Upper y coordinate for local refinement zone.

Figure 6 shows the MESHMAKER input data for a grid that will be used for the test problems presented in section 5.0. With the modifications in the MESHMAKER module, the input file of Figure 6 produces two different mesh files. The first file (MESH) corresponds to a horizontal rectangular coarse grid with 81 grid blocks, 9 in each $\mathrm{X}$ and $Y$ direction and 1 in the $Z$ direction. Each grid block has a size of $60 \mathrm{~m}$ by $60 \mathrm{~m}$ by $15 \mathrm{~m}$ (DX*DY*DZ). The second file, saved with the name REFIN, corresponds to the locally refined grid. The coarse grid is locally refined in two regions: $0<\mathrm{X}<180,0<\mathrm{Y}<180$, and $360<\mathrm{X}<540,360<\mathrm{Y}<540$. Local grid refinement is performed by dividing each of the coarse blocks encountered in the refinement regions into 9 small grid blocks ( 3 is the coefficient of refinement) with dimensions $20 \mathrm{~m} \times 20 \mathrm{~m} \times 15 \mathrm{~m}$. The user can run simulations with the original file MESH (coarse grid) or can change the name of file REFIN to MESH to run simulations with the locally refined grid. The current implementation allows only local refinement for two-dimensional cartesian grids (X-Y 
plane). For detailed information about the input blocks and records the reader is referred to the TOUGH2 user's manuals (Pruess, 1987, 1991b; Pruess et al., 1999) and to Appendix B.

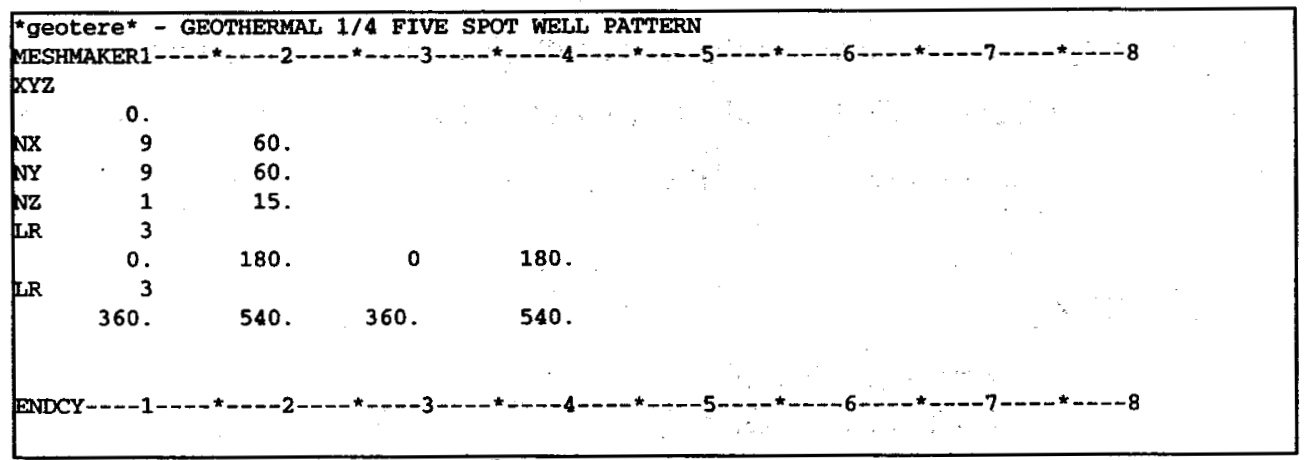

Figure 6

MESHMAKER input for generation of coarse and locally refined grids

\subsection{Numerical Approach and Approximation on Locally Refined Grids}

Having developed a geometric description for locally refined grids, the next step in the implementation is to modify the numerical approach and approximation for locally refined grids in order to be able to accommodate different refinement zones and composite connections. Minor changes to the TOUGH2 subroutine INPUT were made to read and store the new connection data. Due to the existence of interpolation nodes and composite connections, significant changes are required in subroutine MULTI. Subroutine MULTI is responsible for calculating flow-terms, residuals and setting up the coupled linear equations arising at each iteration (Equations 3.3, 3.4 and 3.6). The modifications result in a new scheme that gives better approximation of flow terms across composite connections, and includes into the linear equations the influence of interpolation node reference elements (Jacobian terms).

\subsubsection{Discretization in Locally Refined Grids}

The integral finite difference discretization presented in Section 3.0 applies for both locally refined and uniform grids. Mass flow terms can be approximated using Equation 3.3. However, when two blocks are separated by a composite interface an interpolation 
node is introduced and Equation 3.3 should be modified. To illustrate how flow terms are approximated across composite connections consider the grid shown in Figure 7.

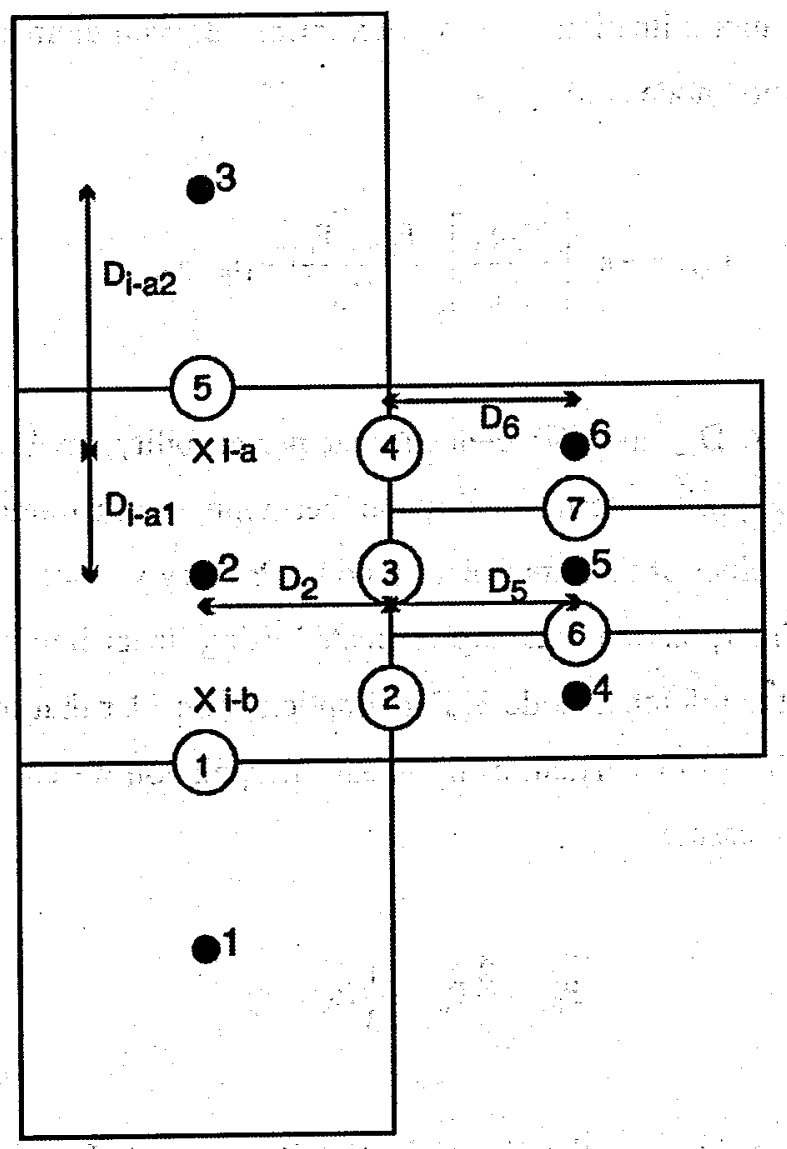

Figure 7

Approximation of flow terms in locally refined grids

Suppose we want to approximate mass flow terms between volume elements 2 and 5 . Equation 3.3 can be applied directly as follows:

$$
F_{\beta, 52}=-k_{52}\left[\frac{k_{r \beta} \rho_{\beta}}{\mu_{\beta}}\right]_{52}\left[\frac{P_{\beta, 5}-P_{\beta, 2}}{D_{52}}-\rho_{\beta, 52} g_{52}\right]
$$

Here the subscripts (52) indicate that the quantities $k_{52},\left[\frac{k_{\beta}}{\mu_{\beta}}\right]_{52}, \rho_{\beta, 52}$ are evaluated at the interface using the actual thermophysical parameters of elements 5 and 2 with a suitable 
averaging technique(e.g., upstream weighting). $D_{52}$ is the distance between nodes 5 and 2 which is equal to $D_{5}+D_{2}$.

Now consider the approximation of mass flux terms between elements 2 and 6 with the use of an interpolation node (i-a):

$$
F_{\beta, 62}=-k_{6 i}\left[\frac{k_{p \beta} \rho_{\beta}}{\mu_{\beta}}\right]_{6 i} \frac{P_{\beta, 6}-P_{\beta, i}}{D_{6 i}}-\rho_{\beta, 6 i} g_{6 i}
$$

Here $D_{6 i}$ is equal to $D_{52}$ and (6i) denotes that permeability, mobility and density are evaluated at the composite interface using the thermophysical parameters of grid block 6 and the estimated values at the interpolation node. Primary variables and thermophysical parameters at the interpolation node are estimated using linear interpolation between the actual node 2 and the reference node 3 . For simplicity consider that the grid blocks 2 and 3 have the same dimensions. Then, using linear interpolation we can write the following expression for the pressure:

$$
P_{\beta, i}=\frac{2}{3} P_{\beta .2}+\frac{1}{3} P_{\beta, 3}
$$

Note that because in this case the Coefficient of Refinement (CR) is 3 the interpolation factors are $2 / 3$ and $1 / 3$. If the coefficient of refinement were for example 5 , the interpolation coefficients would have been $4 / 5$ and $1 / 5$ or $3 / 5$ and $2 / 5$ depending on the location of the interpolation node. Interpolation factors are computed inside subroutine MULTI using the information provided by the enhanced MESH file.

Similarly, in order to approximate heat flux between grid blocks 2 and 6 we use the following equation:

$$
F_{\beta, 62}^{(N K+1)}=-K_{6 i} \frac{T_{6}-T_{i}}{D_{6 i}}+\sum_{\beta=1}^{N P H} h_{6 i} F_{\beta, 6 i}
$$

Finally, note that the interpolation Equation 4.3 is not only applied to the calculation of the pressure at the interpolation nodes, but also for all other primary variables as well as 
all the thermophysical properties (secondary parameters: phase saturation, relative permeability, viscosity, density, specific enthalpy, capillary pressure and temperature).

\subsubsection{Newton-Raphson Iteration}

In order to illustrate the Newton-Raphson iteration approach, consider a non-isothermal one-component simulation with $\mathrm{N}$ grid blocks. Equation 3.6 represents a system of coupled nonlinear equations with $2 \mathrm{~N}$ unknowns (Pressure and Temperature/Saturation). Residuals given by Equation 3.4 are updated in subroutine MULTI once all the flow terms are calculated for a specified connection. Neglecting higher order terms, residuals are linearized using Taylor expansion around the assumed solution $x_{i, p+1}$. Here $x_{i}$ denotes the set of primary variables (two per each grid block). Finally, Newton-Raphson iteration is used to solve for the increments $\mathrm{x}_{\mathrm{i}, \mathrm{p}+1}-\mathrm{x}_{\mathrm{i}, \mathrm{p}}$. Equation 3.6 can be rewritten in a matrix form as:

$$
\left[\begin{array}{ccccc}
\mathbf{J}_{1,1} & \mathbf{J}_{1,2} & \mathbf{J}_{1,3} & \cdots & \mathbf{J}_{1,2 N} \\
\mathbf{J}_{2,1} & \mathbf{J}_{2,2} & \mathbf{J}_{2,3} & \cdots & \mathbf{J}_{2,2 N} \\
\mathbf{J}_{3,1} & \mathbf{J}_{3,2} & \mathbf{J}_{3,3} & \cdots & \mathbf{J}_{3,2 N} \\
\vdots & \vdots & \vdots & \ddots & \vdots \\
\mathbf{J}_{2 N, 1} & \mathbf{J}_{2 \mathrm{~N}, 2} & \mathbf{J}_{2 \mathrm{~N}, 3} & \cdots & \mathbf{J}_{2 \mathrm{~N}, 2 \mathrm{~N}}
\end{array}\right] \times\left[\begin{array}{c}
\Delta \mathbf{x}_{1} \\
\Delta \mathbf{x}_{2} \\
\Delta \mathbf{x}_{3} \\
\vdots \\
\Delta \mathbf{x}_{2 N}
\end{array}\right]=\left[\begin{array}{c}
-\mathbf{R}_{1} \\
-\mathbf{R}_{2} \\
-\mathbf{R}_{3} \\
\vdots \\
-\mathbf{R}_{2 N}
\end{array}\right]
$$

[J] symbolizes the Jacobian submatrices, [ $\Delta \mathbf{x}]$ the vector of unknown primary variables and [R] the vector of residuals for each grid block. The diagonal submatrices $\mathbf{J}_{n, n}$ represent according to Equation 3.6 the partial derivatives of the residuals in element $n$ with respect to the primary variables in the same element. Whereas, the off-diagonal submatrices $J_{m, n}$ carry the partial derivatives of the residual in element $m$ with respect to the primary variables in element $n$ (i.e, Jacobian terms due to flow terms). All the residuals and therefore their partial derivatives are related to the flow terms through Equation 3.4. For instance, off-diagonal Jacobian terms can be calculated as follows:

$$
-\frac{\partial R_{n}^{(k) k+1}}{\partial x_{i}}=-\frac{\Delta t}{V_{n}} A_{n m} \frac{\partial F_{m}^{(k) k+1}}{\partial x_{i}}
$$


Using Equation 4.6 subroutine MULTI computes the off-diagonal Jacobian sub-matrices $\mathbf{J}_{\mathrm{m}}$ inside a loop over connections. In the conventional approach each regular connection between volume elements $m$ and $n$ generates two off-diagonal Jacobian submatrices. Elements not connected produce an empty or zero submatrix. However, for composite connections, the flow between two elements $m$ and $n$ does not depend only on the primary variables of the respective elements but also on the primary variables of a reference element $l$. As discussed before, a reference node is required to obtain an approximation of primary variables and thermophysical parameters at the fictitious interpolation node. The implication is that two additional Jacobian submatrices are required with off-diagonal locations corresponding to the intersection of the two grid blocks connected by the composite connection ( $m$ and $n$ ) and the grid block corresponding to the reference element $(l)$.

Now, let us turn our attention to the linear equation setup as it is currently implemented in TOUGH2. All Jacobian terms are stored sequentially in a one-dimensional array CO. The corresponding row and column indices are stored separately in arrays IRN and ICN, respectively. The first Jacobian elements to be stored are those arising from accumulation terms. Jacobian elements obtained from flow terms are subsequently assigned to the array CO. Some of the additional Jacobian terms created due to the presence of composite connections will occur in matrix locations previously occupied by other Jacobian terms.

Figure 8 illustrates how the linear equation setup is performed for the grid in Figure 7. For simplicity we will consider only one equation per grid block. Note the multiple entries for locations $(2,1),(2,3)$. These and the Jacobian terms in location $(4,1)$ and $(6,3)$ are due to the presence of composite connections and are shown in bold type.

Another important issue to consider is the calculation of all the fluxes required for computing the partial derivatives (Equation 4.6). At each interface we have one flux for each component (sum of contributions from all phases). To compute the partial derivatives we need also the fluxes obtained by incrementing each of the primary variables.(NEQ) for the "first" element, and each of the primary variables (NEQ) for the "second" element. So far the number of required fluxes is $2 * N E Q+1$. However, when the connection is composite we need also the fluxes obtained by incrementing each primary variable for the "reference" element. These connections are recognized by subroutine 
MULTI because their interface area is set to a negative value; the total number of fluxes that need to be calculated is then $3 * \mathrm{NEQ}+1$.

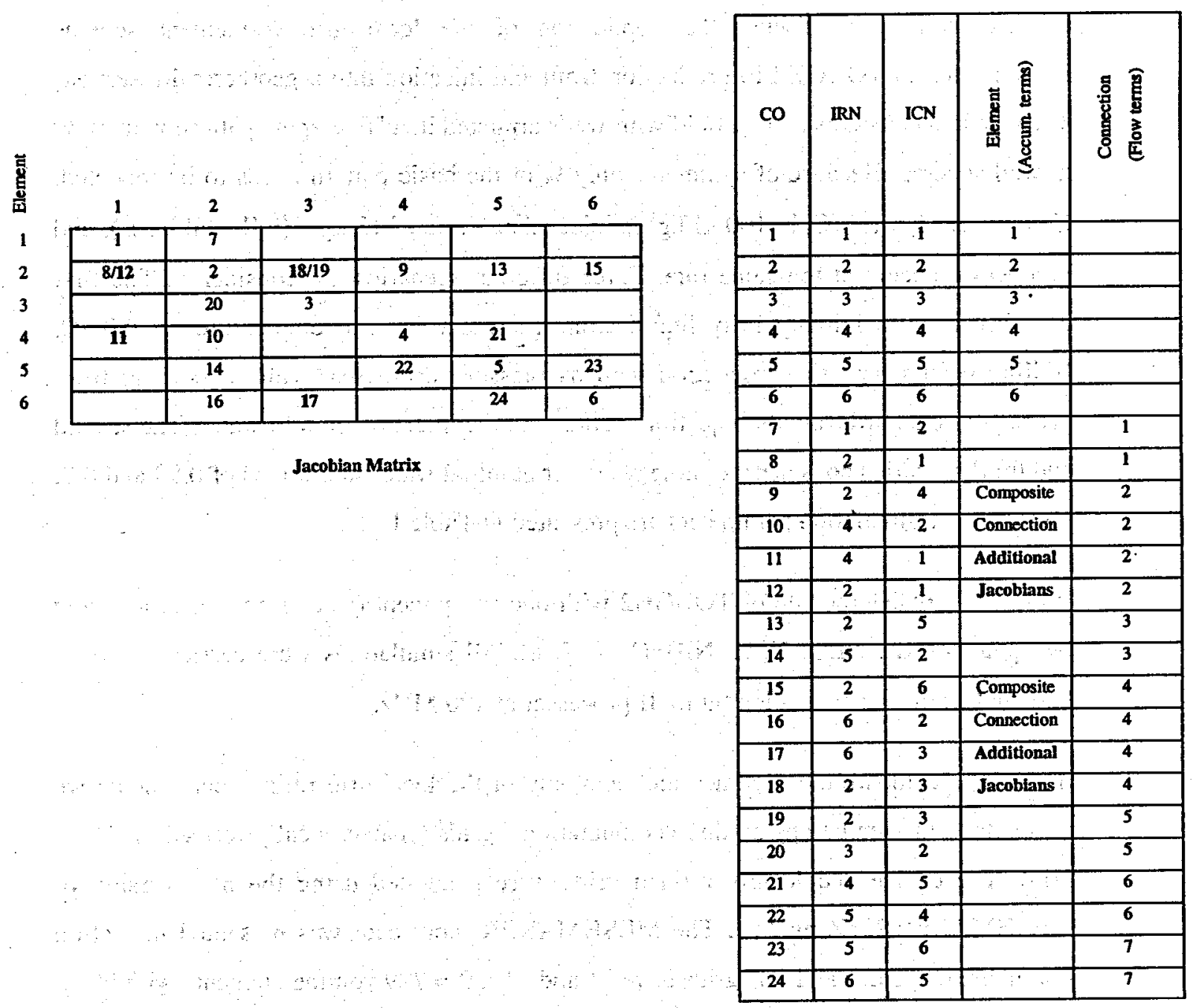

Figure 8

Linear Equation Setup 
In this section we discuss the application of the local grid refinement scheme implemented in TOUGH2 to production from and injection into a geothermal reservoir. The problem considers a large field with wells arranged in a "five-spot" pattern with 1080 $\mathrm{m}$ well spacing. Because of symmetry only $1 / 4$ of the basic pattern needs to be modeled. Cold water $\left(\mathrm{T} \approx 21.3^{\circ} \mathrm{C}, \mathrm{h}=100 \mathrm{~kJ} / \mathrm{kg}\right.$ ) is injected at a rate of $15 \mathrm{~kg} / \mathrm{s}$ (full-well basis), and production occurs at the same rate. Three different scenarios are considered. The first case involves an initially fully liquid-saturated reservoir at a temperature of $250^{\circ} \mathrm{C}$. Boiling occurs near the production well as pressure decreases, while a cooling front propagates outward from the injection well due to the injection of cold water. The second and third scenarios consider the same reservoir at initial water saturations of 0.99 and 0.9 , respectively. Simulation parameters are presented in Table 1.

We use the EOS1 module of TOUGH2 with one component (water), two equations and two phases $(\mathrm{NK}, \mathrm{NEQ}, \mathrm{NPH}, \mathrm{NB})=(1,2,2,6)$. All simulations were carried out on a Personal Computer with a Pentium ${ }^{\circledR}$ II processor at $400 \mathrm{MHz}$.

In order to compare the accuracy and economy of the local grid refinement scheme we conducted our simulations on three computational grids (coarse, locally refined, and fine grid). The coarse and locally refined grids were generated using the new version of MESHMAKER/XYZ module. The MESHMAKER input data was presented in section 4.0 Figure 6. Coarse and fine grids have 81 and $81 \times 9=729$ volume elements with block

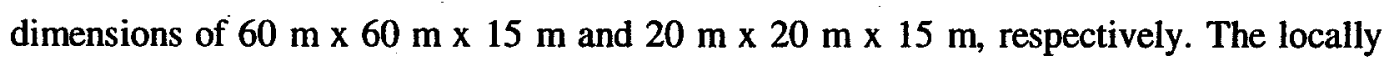
refined grid is composed of 225 volume elements with two different grid block sizes corresponding to the coarse and fine grid block dimensions (See Figure 9). 
(6.6.5.40)

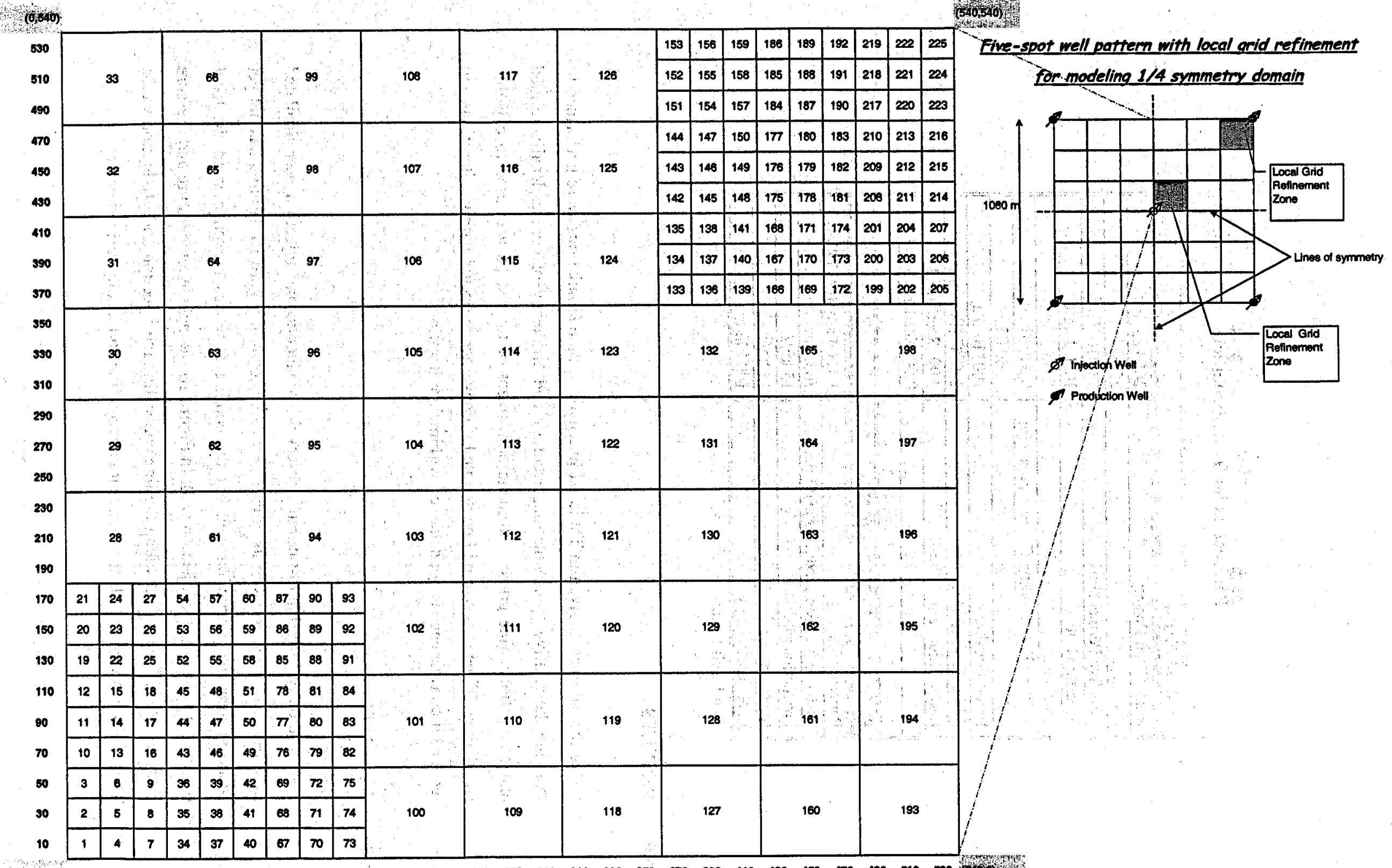

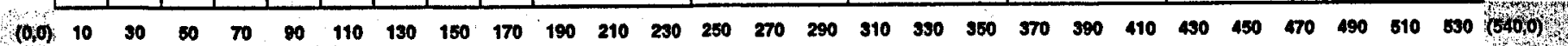

Figure 9. Five-spot well pattern with local grid refinement for modeling 1/4 symmetry domain 
Table 1

Parameters for Test Simulations

\begin{tabular}{|l|l|}
\hline FORMATION & \\
\hline Rock Grain Density & $2650 \mathrm{~kg} / \mathrm{m}^{3}$ \\
\hline Porosity & $25 \%$ \\
\hline Effective Permeability & $1.0^{*} 10^{-13} \mathrm{~m}^{2}$ \\
\hline Specific Heat & $1000 \mathrm{~J} / \mathrm{kg}^{\circ} \mathrm{C}$ \\
\hline Thickness & $15 \mathrm{~m}$ \\
\hline Relative Permeability: Corey curves with & $\mathrm{S}_{\mathrm{r}}=0.30$ \\
\hline & $\mathrm{S}_{\mathrm{g}}=0.05$ \\
\hline Initial Pressure & $100^{*} 10^{5} \mathrm{~Pa}$ \\
\hline Initial Temperature & $250^{0} \mathrm{C}($ Scenario 1$)$ \\
\hline Initial Liquid Saturation & $0.99($ Scenario 2) \\
\hline & $0.90(\mathrm{Scenario} 3)$ \\
\hline & \\
\hline PRODUCTION/NJECTION & \\
\hline Pattern Area & $1.1664 \mathrm{~km}{ }^{2}\left[(2 * 540 \mathrm{~m})^{2}\right]$ \\
\hline Distance between producers and injectors & $763.7 \mathrm{~m}$ \\
\hline Production rate( $\mathrm{(a)}$ & $15 \mathrm{~kg} / \mathrm{s}$ \\
\hline Injection rate ${ }^{(a)}$ & $15 \mathrm{~kg} / \mathrm{s}$ \\
\hline Injection enthalpy & $100 \mathrm{~kJ} / \mathrm{kg}$ \\
\hline
\end{tabular}



\subsection{RESULTS}

Using the parameters given in Table 1 ( 3 scenarios), nine simulations were performed to examine the relative approximation, accuracy and efficiency of coarse, locally refined and conventional fine grids. Automatic adjustment of time steps was used to expedite simulations. Time step size will be doubled if convergence occurs within 4 iterations and reduced if convergence is not achieved within eight iterations.

\subsection{Scenario \#1}

For an initially fully liquid-saturated reservoir, production and injection was simulated for a period of 10 years. Results after 2 years are shown in Figure 10. Boiling has not yet occurred near the production well but the cooling front has moved beyond the local refinement zone near the injection well. In Figure 10 we include temperature and pressure contours as well as profiles along the line connecting the injection and production wells. The results show an excellent agreement between fine and locally refined grids. 

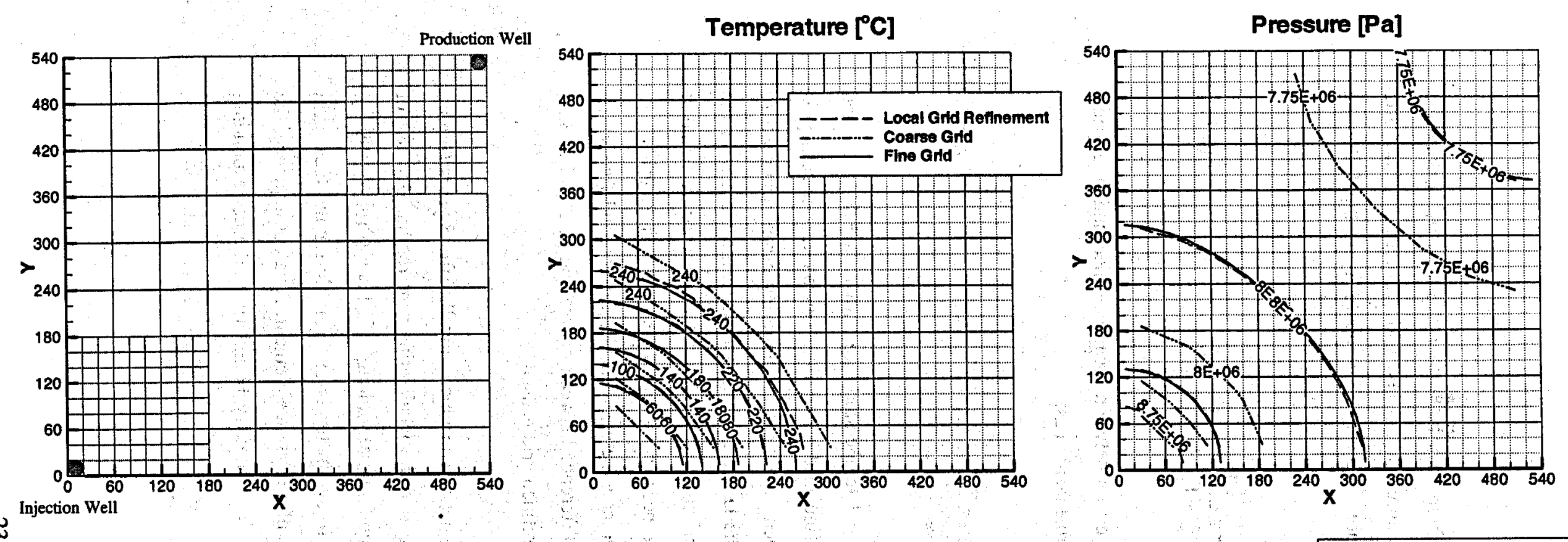
W
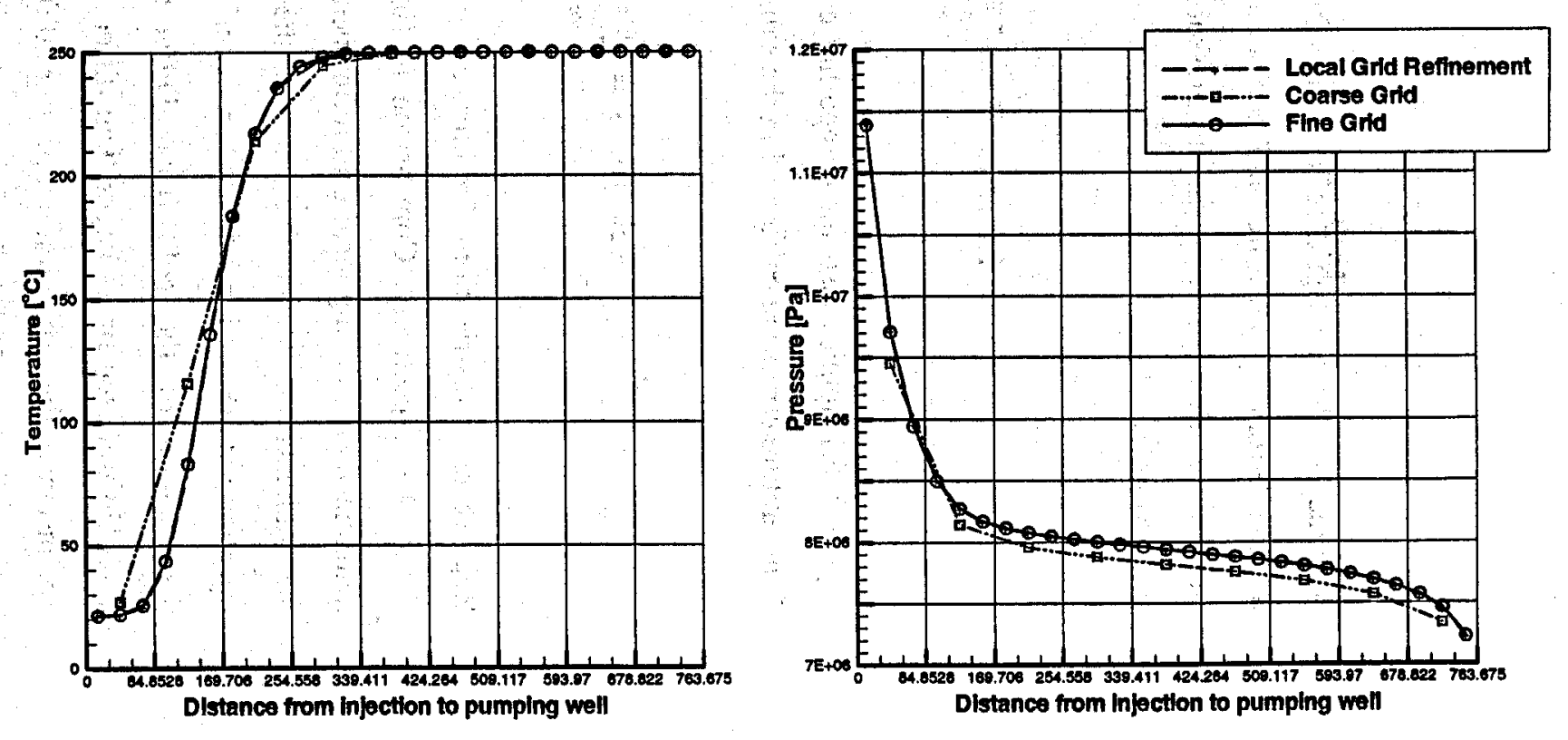

Figure 10

Temperature and pressure comparison for fine, coarse and locally refined grids - Scenario \# 1 - Time $=2$ years 
Accuracy of the local grid refinement scheme can be further explored by considering relative errors of pressure and temperature increments with respect to fine grid simulations. Relative errors in the pressure field can be compute using the following expression:

$$
\text { Relerror_P }=\frac{\mathbf{P}_{\text {coarse }}-P_{\text {fine }}}{P_{\text {fine }}-P_{\text {initial }}}-1
$$

Equation 6.1 applies also for computing errors between locally refined and fine grids. Temperature errors are calculated in the same fashion as:

$$
\text { Relerror_T }=\frac{T_{\text {coarse }}-T_{\text {fine }}}{T_{\text {fine }}-T_{\text {initial }}}-1
$$

Relative error distributions for pressure and temperature are shown in Figure 11 and Figure 12 respectively. Comparison to fine grid simulations shows that accuracy is maintained when using local grid refinement. As expected the larger errors occur where the gradients are steeper. For pressure, the larger gradients occur in the vicinity of the injection well (See Figure 10), whereas for temperature large relative errors are found at the location of the cold front. Differences between coarse and fine grids are approximately ten times larger than differences between locally refined and fine grids. For instance, the maximum temperature difference between coarse and fine grid is $\mathbf{3 2 . 5}$ ${ }^{\circ} \mathrm{C}$, compared to $4.28^{\circ} \mathrm{C}$ when the locally refined grid is used.

As time elapses, boiling starts taking place near the production well. After 8 years (Figure 13) the phase front has already moved beyond the local refinement region. Because of grid orientation effects, the front moves preferentially along grid lines (Pruess, 1991a). The presence of no flow boundaries due to symmetry is unfavorable for the local grid refinement scheme because of the absence of a reference element. As a consequence, the scheme loses accuracy and behaves near the symmetry boundary as the corresponding coarse grid. 

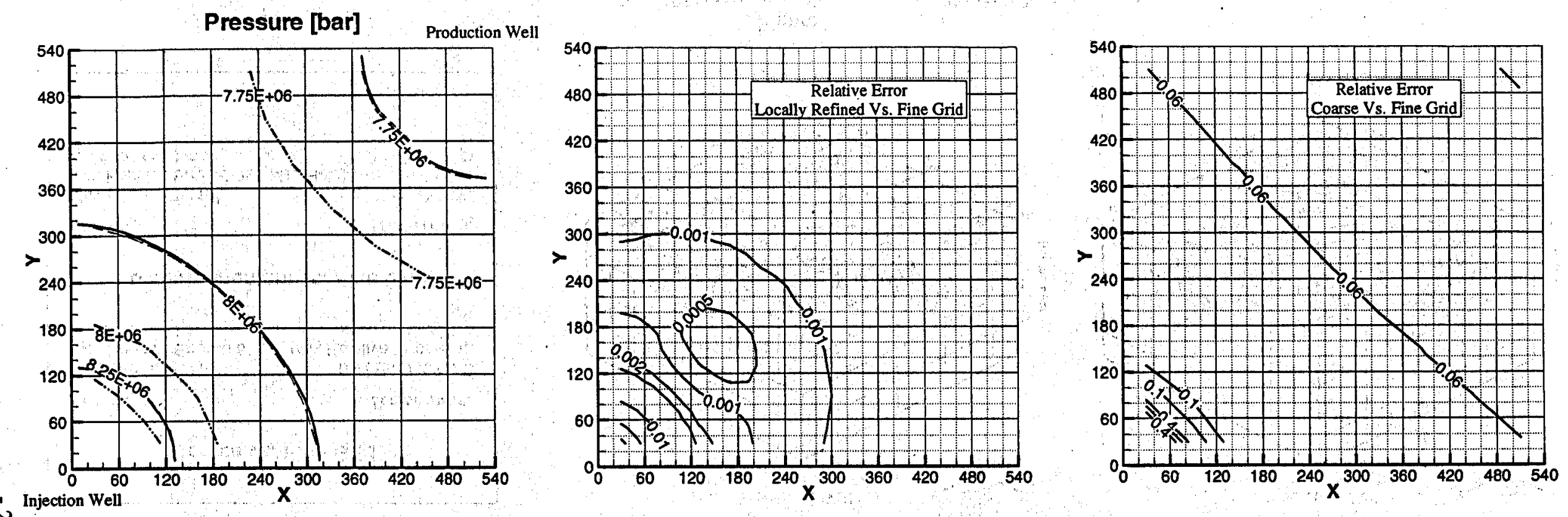

出
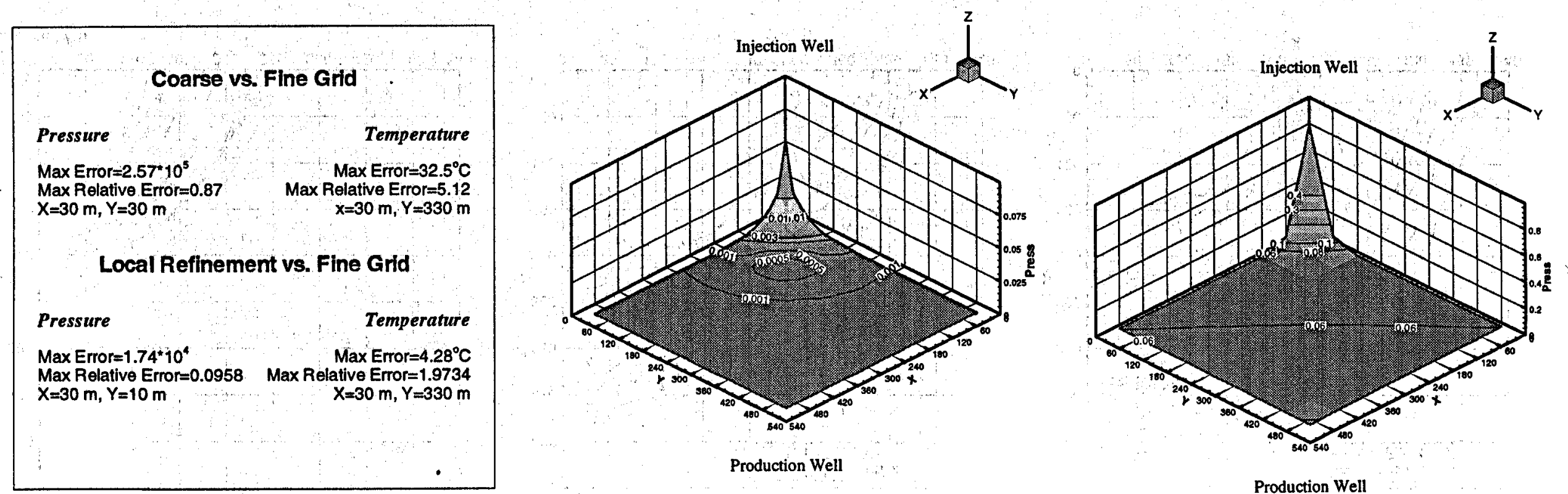

Figure 11

Relative Error Pressure - Scenario \# 1 - Time: 2 Years 

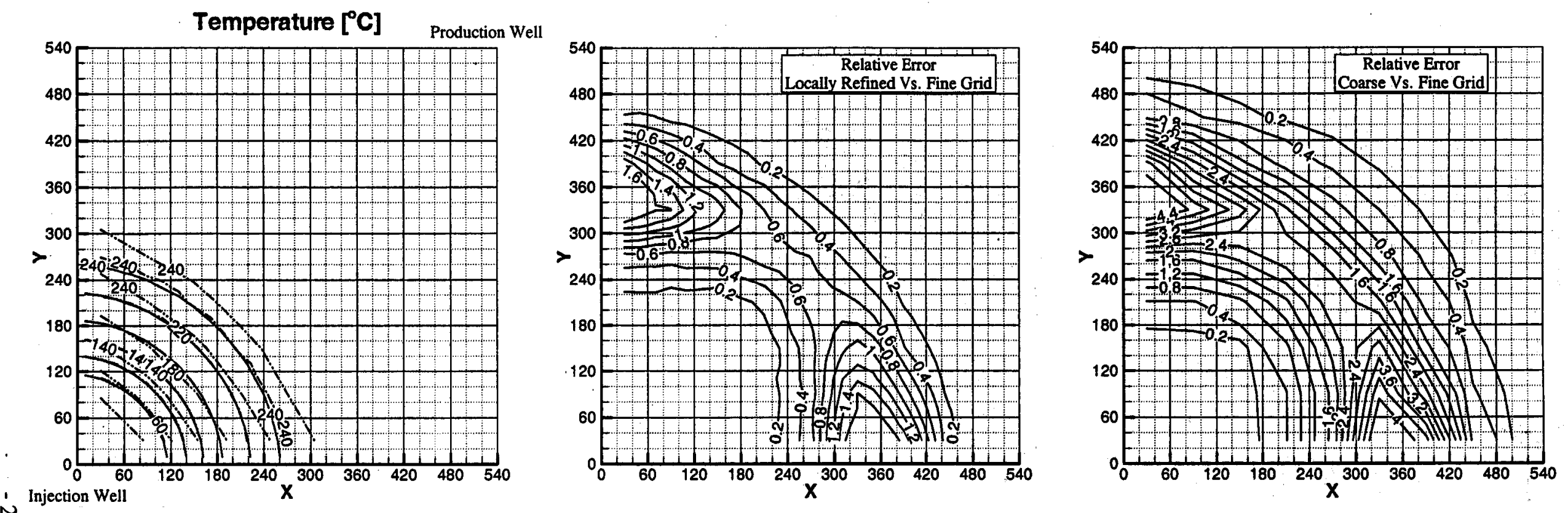

ส


. Figure 12

Relative Error Temperature - Scenario \# 1 - Time: 2 Years 


\section{Gas Saturation}

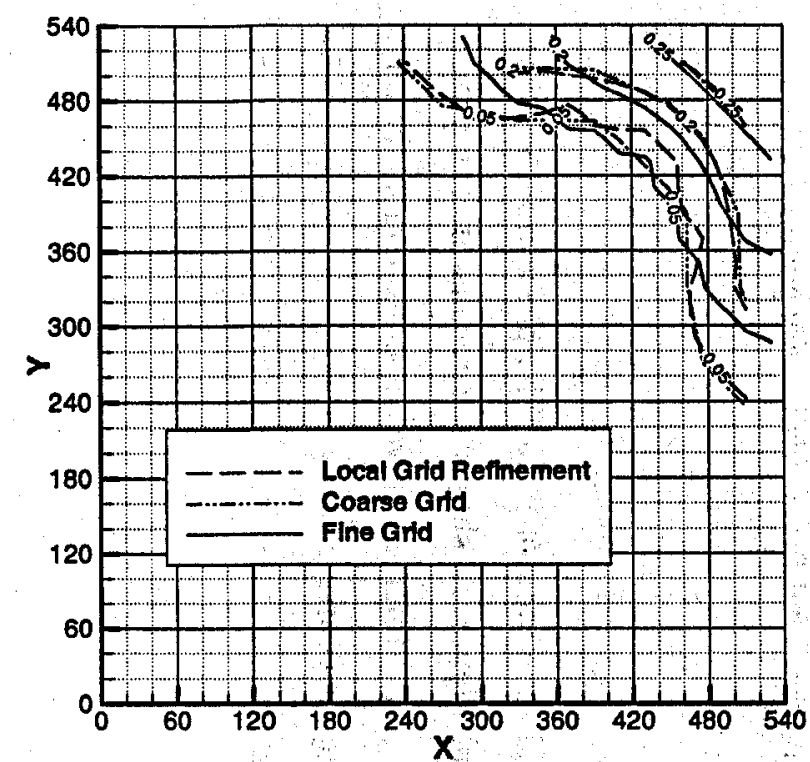

Ñ

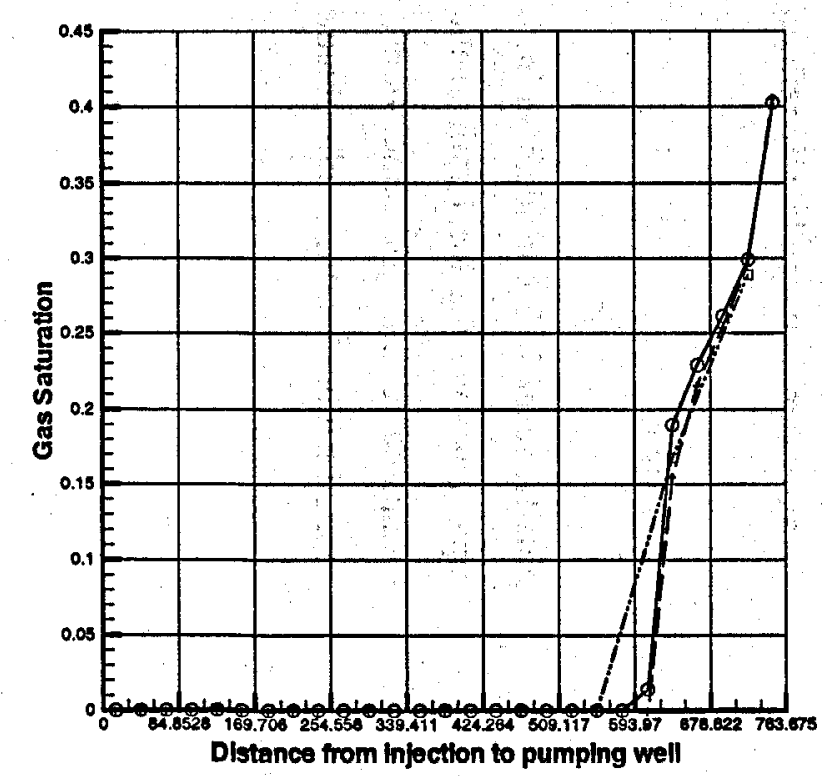

Temperature [ ${ }^{\circ} \mathrm{C}$ ]


Pressure [Pa]


Saturation, temperature and pressure - Scenario \# 1 - Time $=8$ years 
Evolution of saturation, pressure and temperature with time for 3 points along the line of injection-production wells is presented in Figures 14,15 and 16. The first point is selected in the middle of the refinement region near the injection well $(X=90 \mathrm{~m}, Y=90$ $\mathrm{m}$ ). The second point is located in the middle of the domain (coarse region, $X=270 \mathrm{~m}, \mathrm{Y}$ $=270 \mathrm{~m}$ ). Finally the third point is located in the middle of the refinement region corresponding to the production well $(X=450 \mathrm{~m}, Y=450 \mathrm{~m})$. A good agreement between locally refined grid simulations and fine grid results is obtained for the first 4 years. After the fifth year, temperature and saturation gradients are concentrated in the middle of the domain (Coarse region in locally refined grids). At this point, the local grid refinement scheme does not provide additional advantages from coarse grid simulations.

\subsection{Scenario \#2}

Scenario \# 2 considers the geothermal reservoir described in section 5.0 with an initial liquid saturation of $99 \%\left(S_{1}=0.99\right)$. Because of the injection of cold water, condensation around the injection well propagates rapidly. Figure 17 shows the results after 1 year. Fine and locally refined grids produce similar results, whereas coarse grid results show an inferior performance for temperature and pressure approximation.

After the second year (Figure 18) the saturation (boiling) front moves to the coarse region (middle of the domain). The local grid refinement scheme starts losing accuracy, yet it provides a better approximation than the results obtained using the coarse grid. Figures 19, 20, and 21 show the evolution of saturation, pressure and temperature for three locations along the line between injection and production wells. For the first two years the agreement between locally refined and conventional fine grids is very good.

\subsection{Scenario \#3}

Finally, scenario 3 considers an initial liquid saturation of $90 \%$. The phase front moves rapidly beyond the local refinement region. Figure 22 shows the results after 1 year. Compared to fine grid simulations, local grid refinement gives a considerably better approximation than the results obtained using the coarse grid. 
Saturation $(X=90 \mathrm{~m}, \mathrm{Y}=90 \mathrm{~m})$
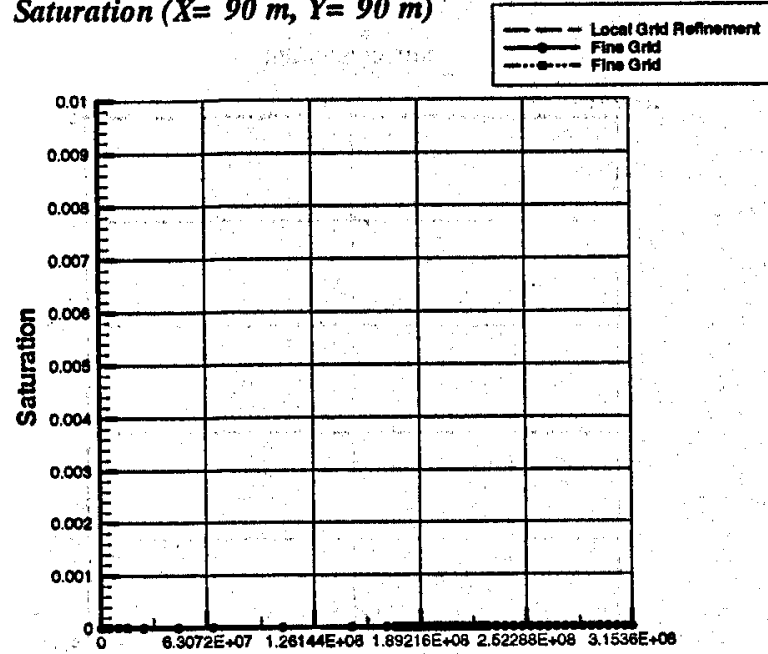

Simulation time

No
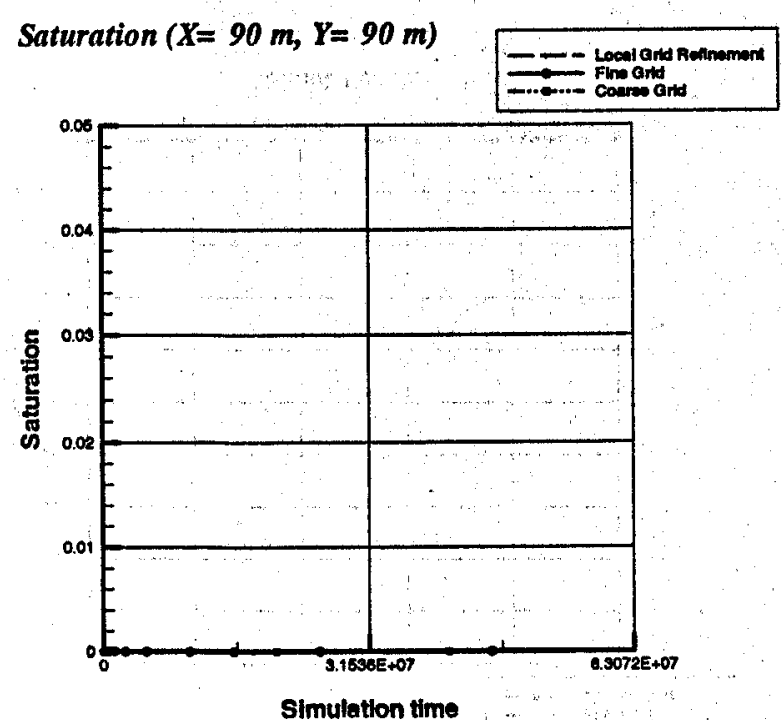

Pressure $(X=90 \mathrm{~m}, \mathrm{Y}=90 \mathrm{~m})$

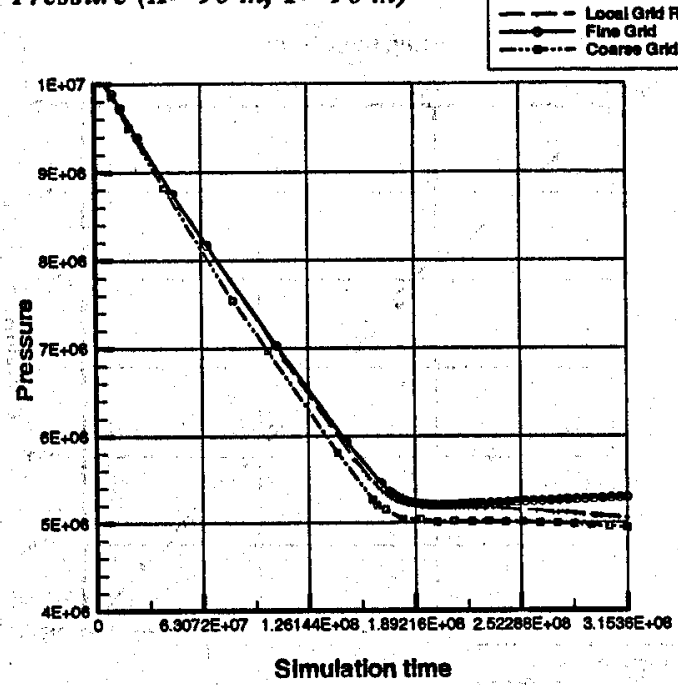

First 6 Years of Simulation

Pressure $(X=90 \mathrm{~m}, Y=90 \mathrm{~m})$

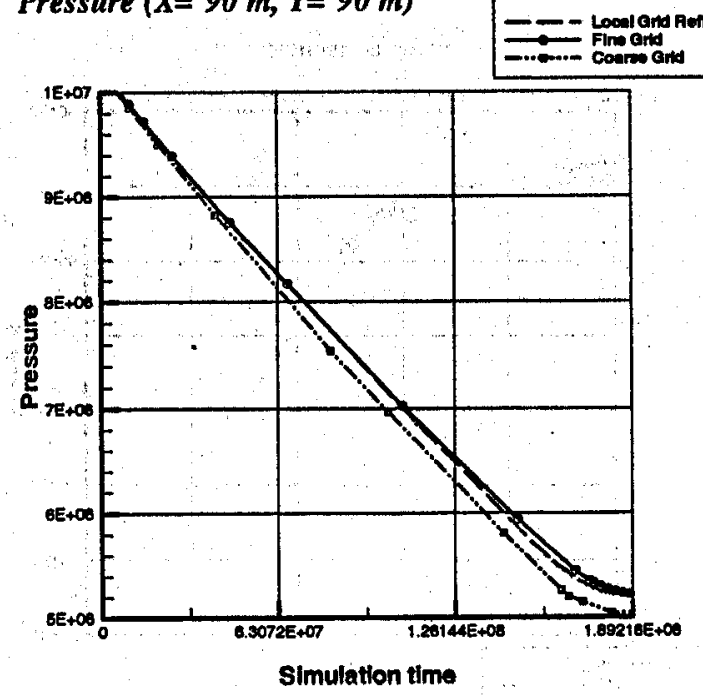

Temperature $(X=90 \mathrm{~m}, Y=90 \mathrm{~m})$
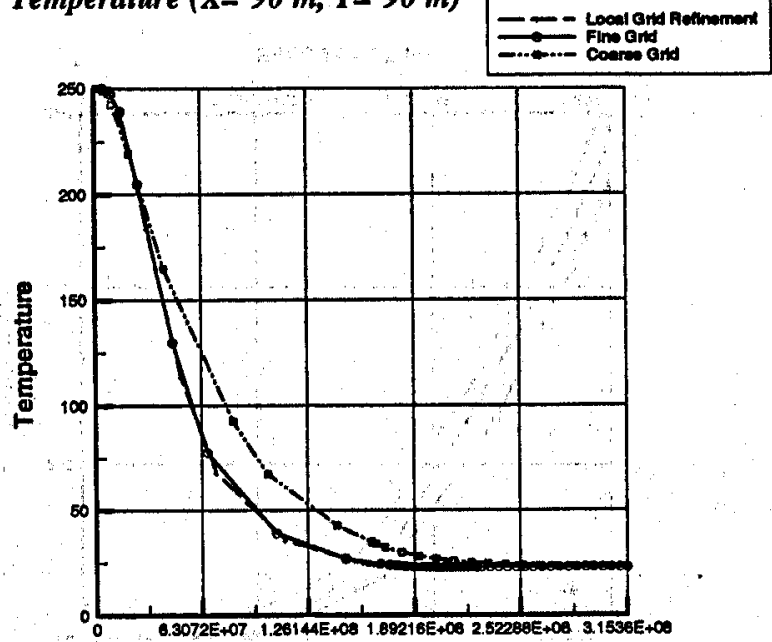

Simulation time
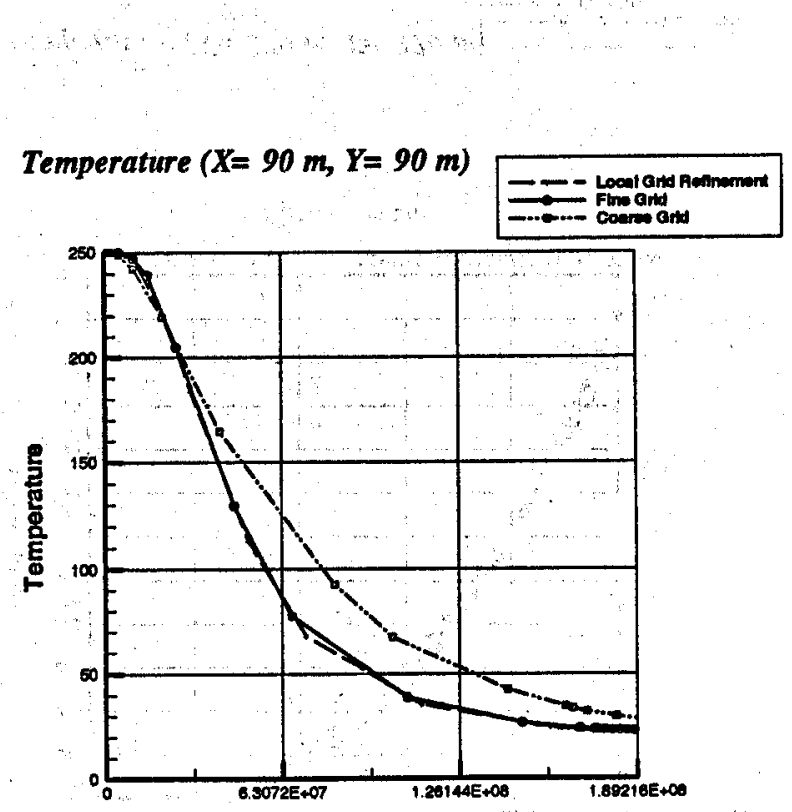

Simulation time

Figure 14

Saturation, Pressure and Temperature at a point near the injection well $(X=90 \mathrm{~m}, \mathrm{Y}=90 \mathrm{~m})$ - Scenario \# 1 


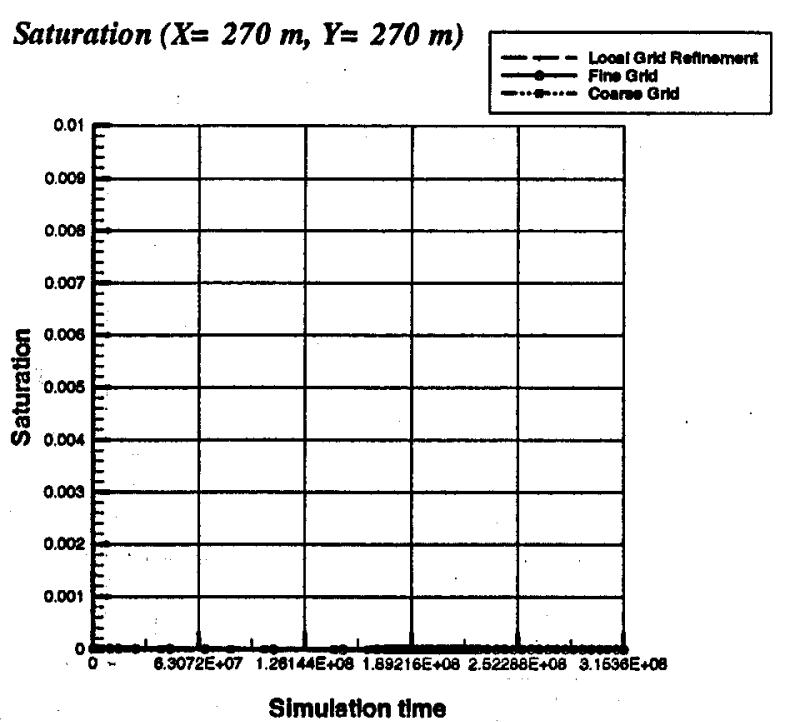

$\dot{\mathscr{o}}$


Pressure $(X=270 \mathrm{~m}, Y=270 \mathrm{~m})$



First 6 years of Simulation

Pressure $(X=270 \mathrm{~m}, Y=270 \mathrm{~m}$ )
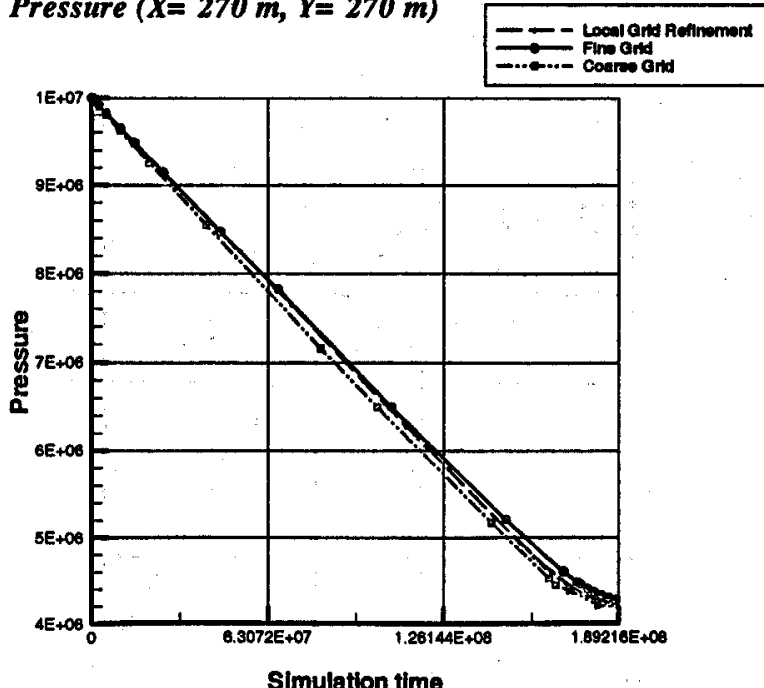

Temperature $(X=270 \mathrm{~m}, Y=270 \mathrm{~m}$



Temperature $(X=270 \mathrm{~m}, Y=270 \mathrm{~m}$
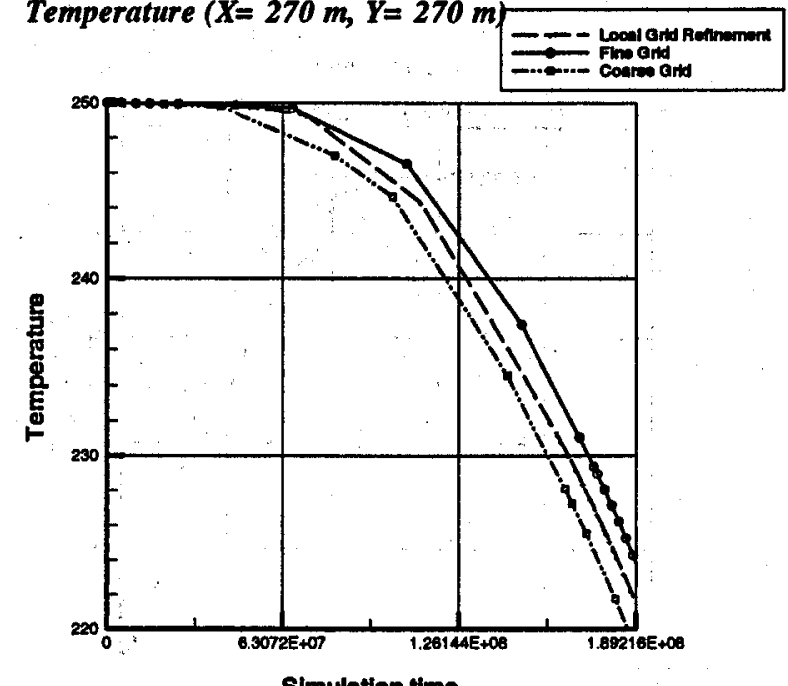

Figure 15

Saturation, Pressure, and Temperature at the middle of the domain $(X=270 \mathrm{~m}, \mathrm{Y}=270 \mathrm{~m})$ - Scenario \# 1 


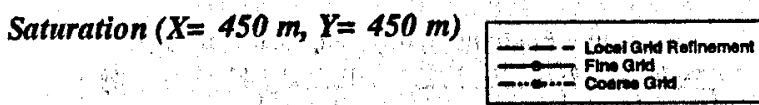

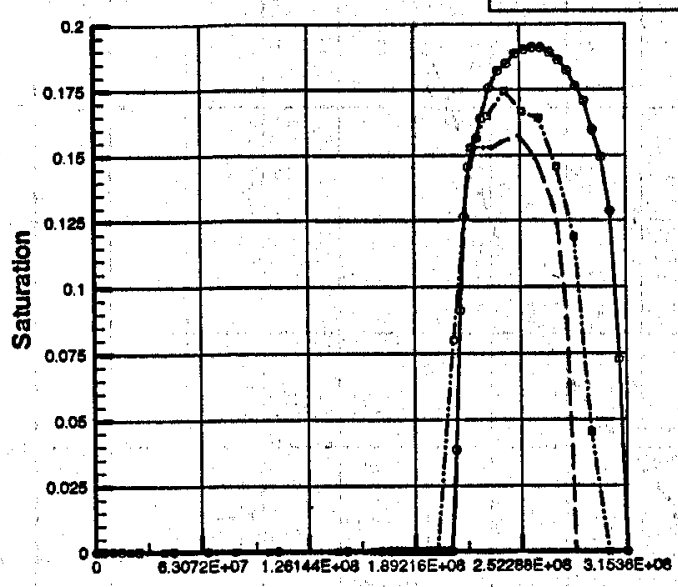

Simulation time

$\stackrel{\omega}{\omega}$
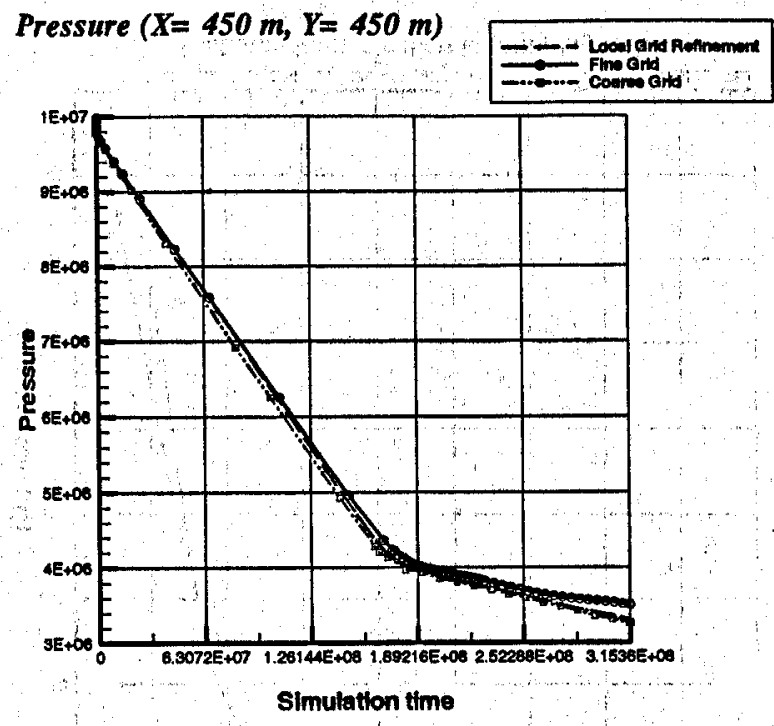

Pressure $(X=450 \mathrm{~m}, \mathrm{Y}=450 \mathrm{~m})$
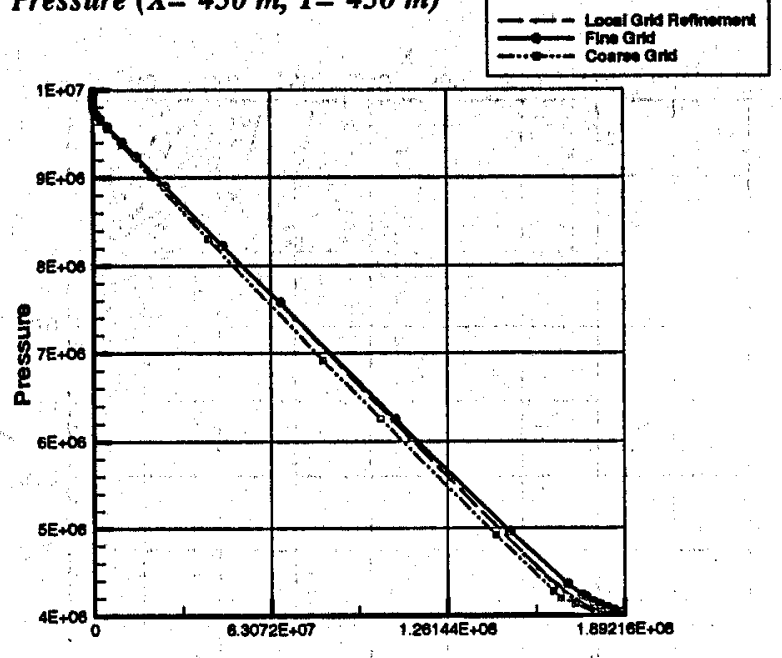

simulation time


Figure 16

Saturation, Pressure, and Temperature at a Point Near the Production Well (X= $450 \mathrm{~m}, \mathrm{Y}=450 \mathrm{~m})$ - Scenario \# 1 


\section{Gas Saturation}

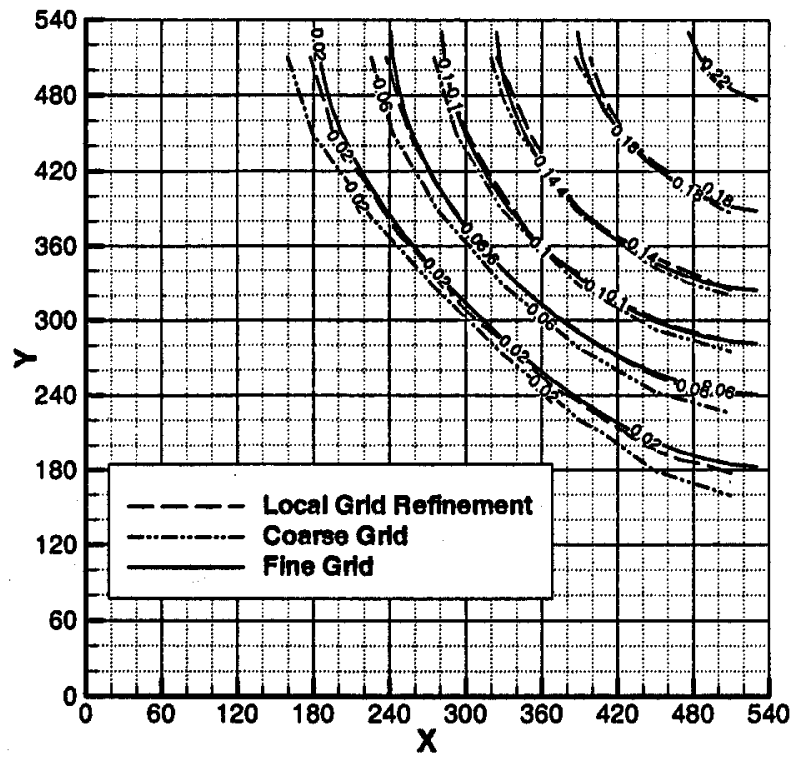

'

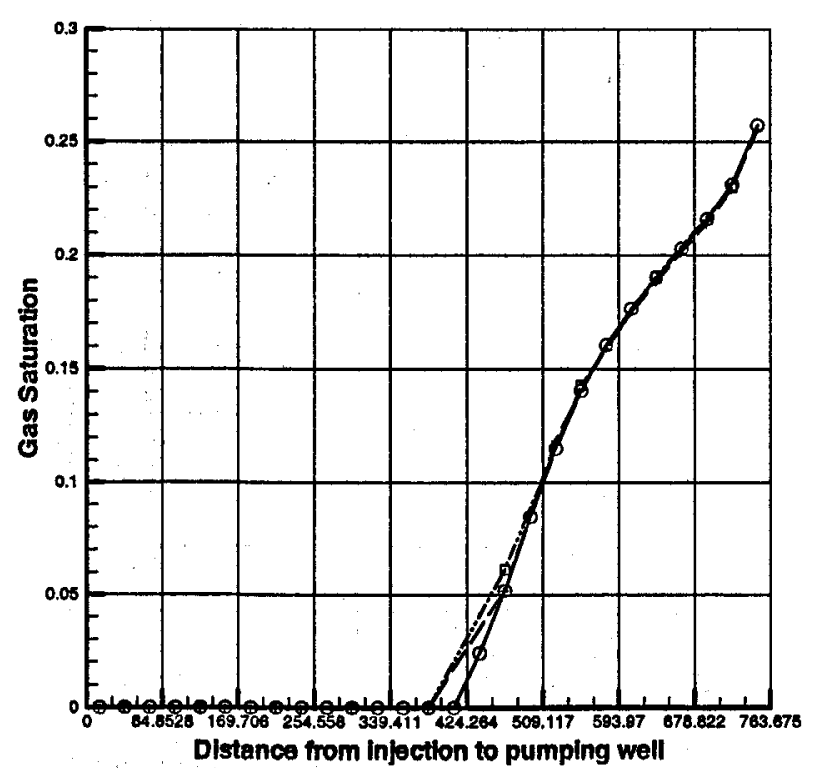

Temperature $\left[{ }^{\circ} \mathrm{C}\right]$
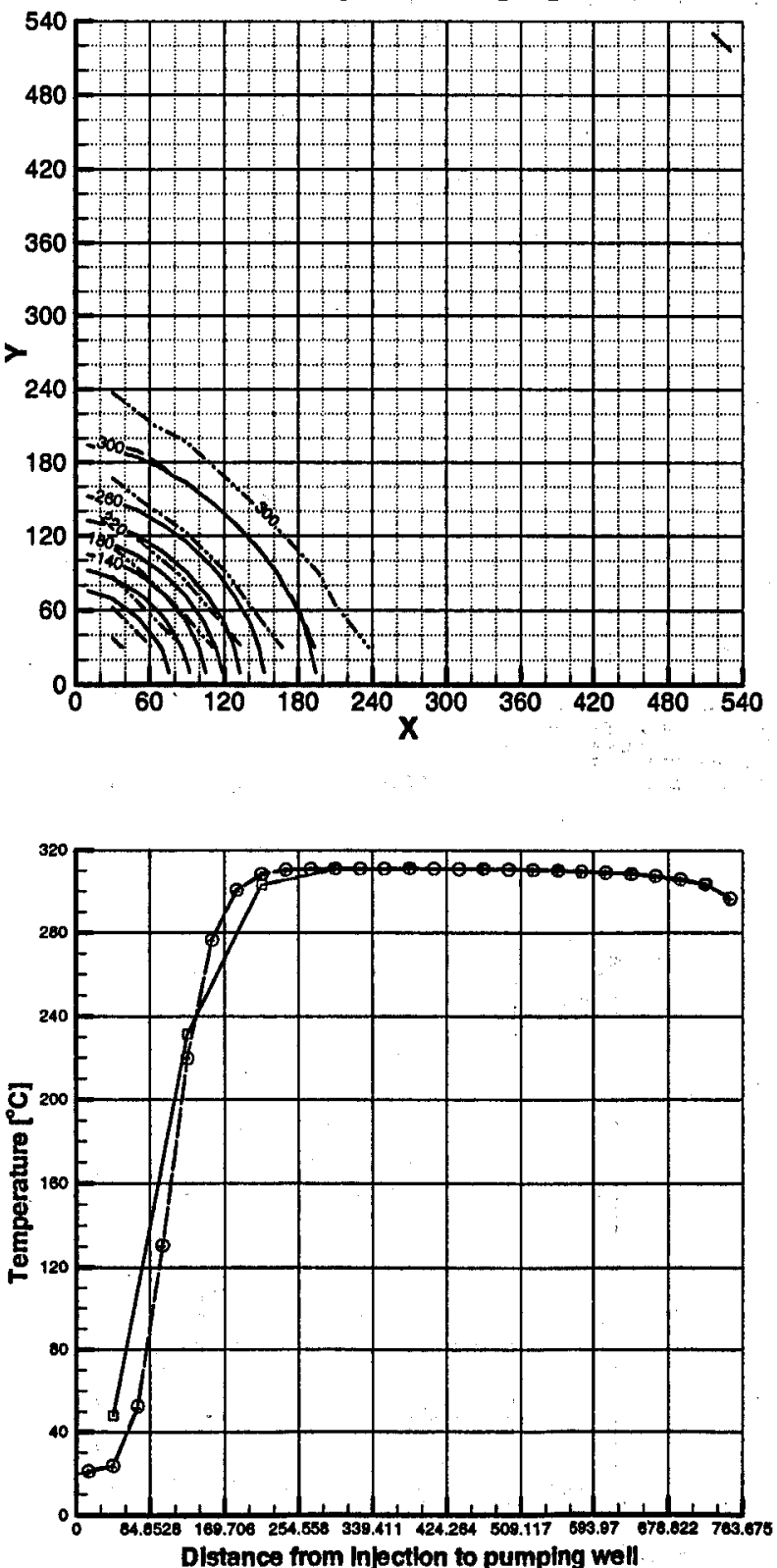

Pressure [Pa]
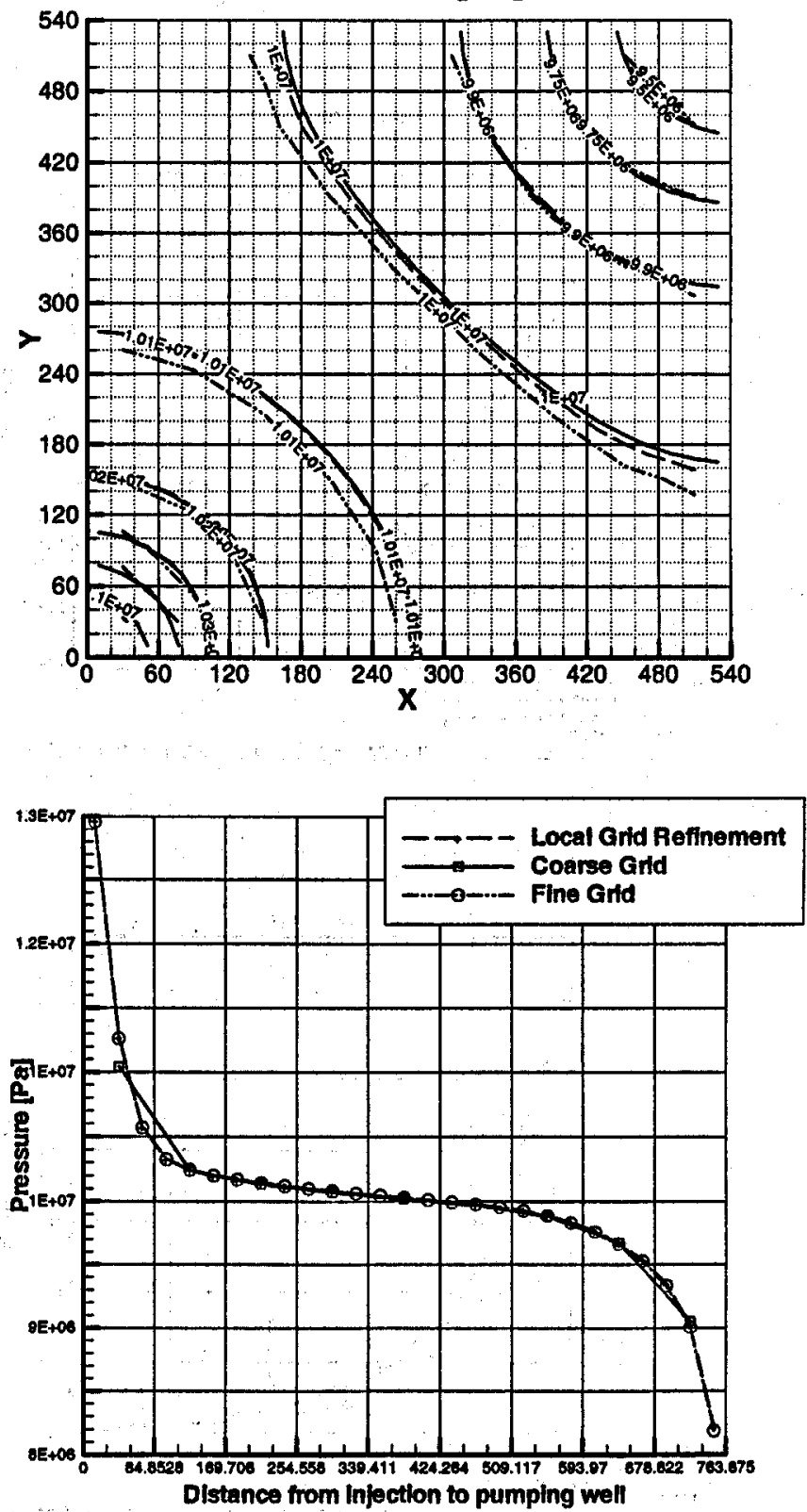

Figure 17

Saturation, temperature, pressure - Scenario \# 2 - Time $=1$ year 
Gas Saturation

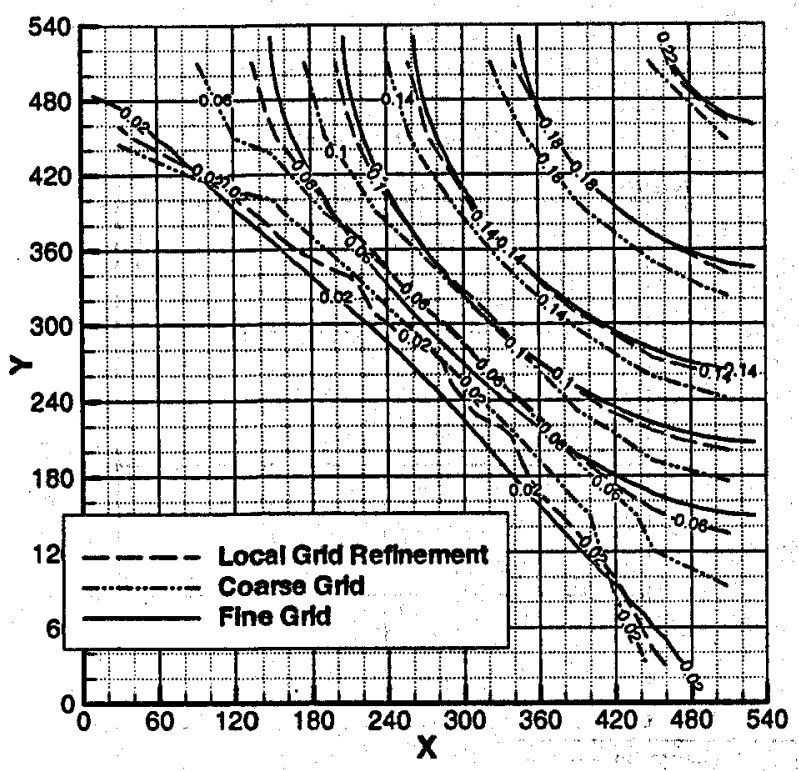

$\dot{w}$



Temperature [ ${ }^{\circ} \mathrm{C}$ ]
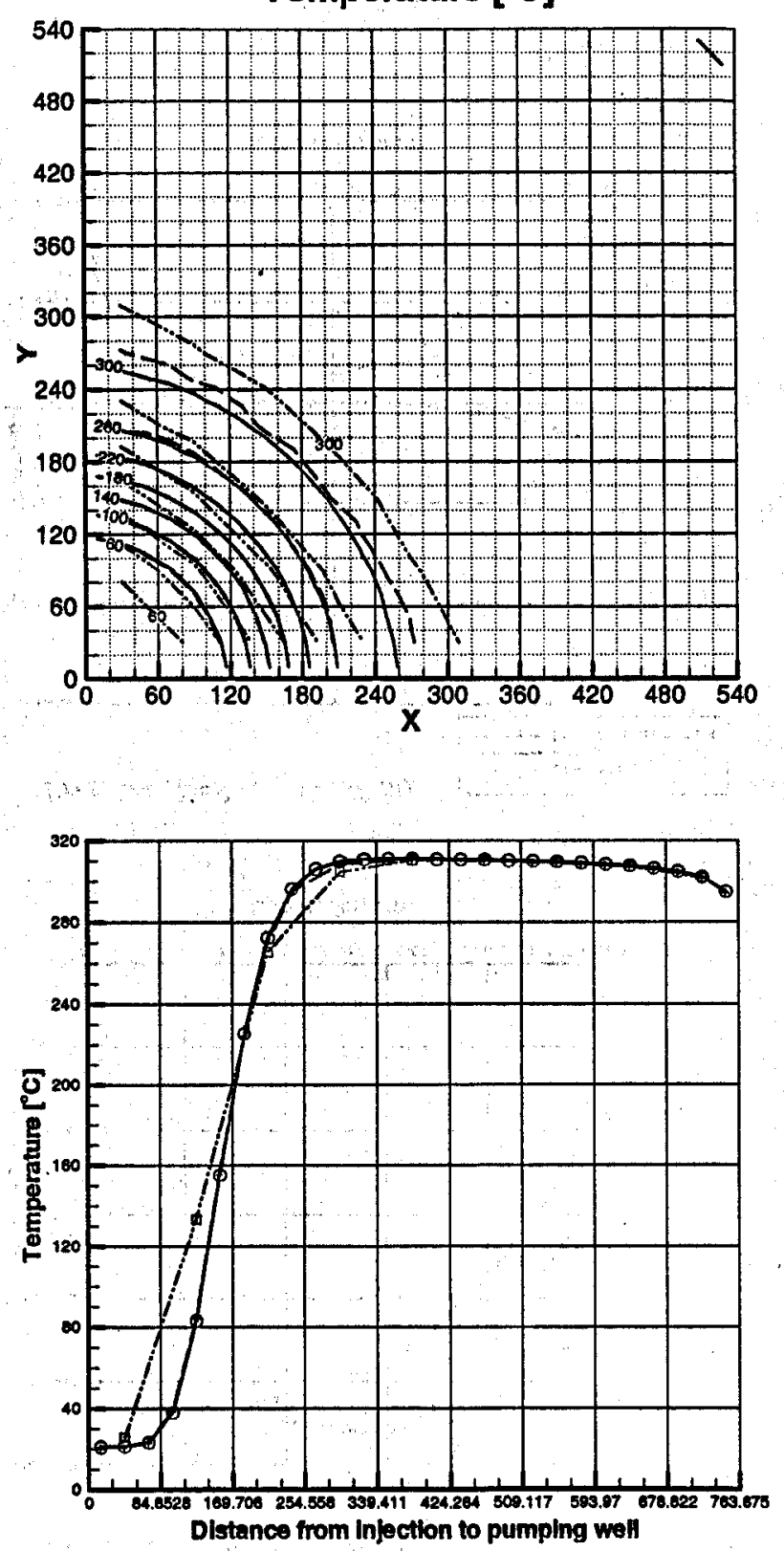

Figure 18
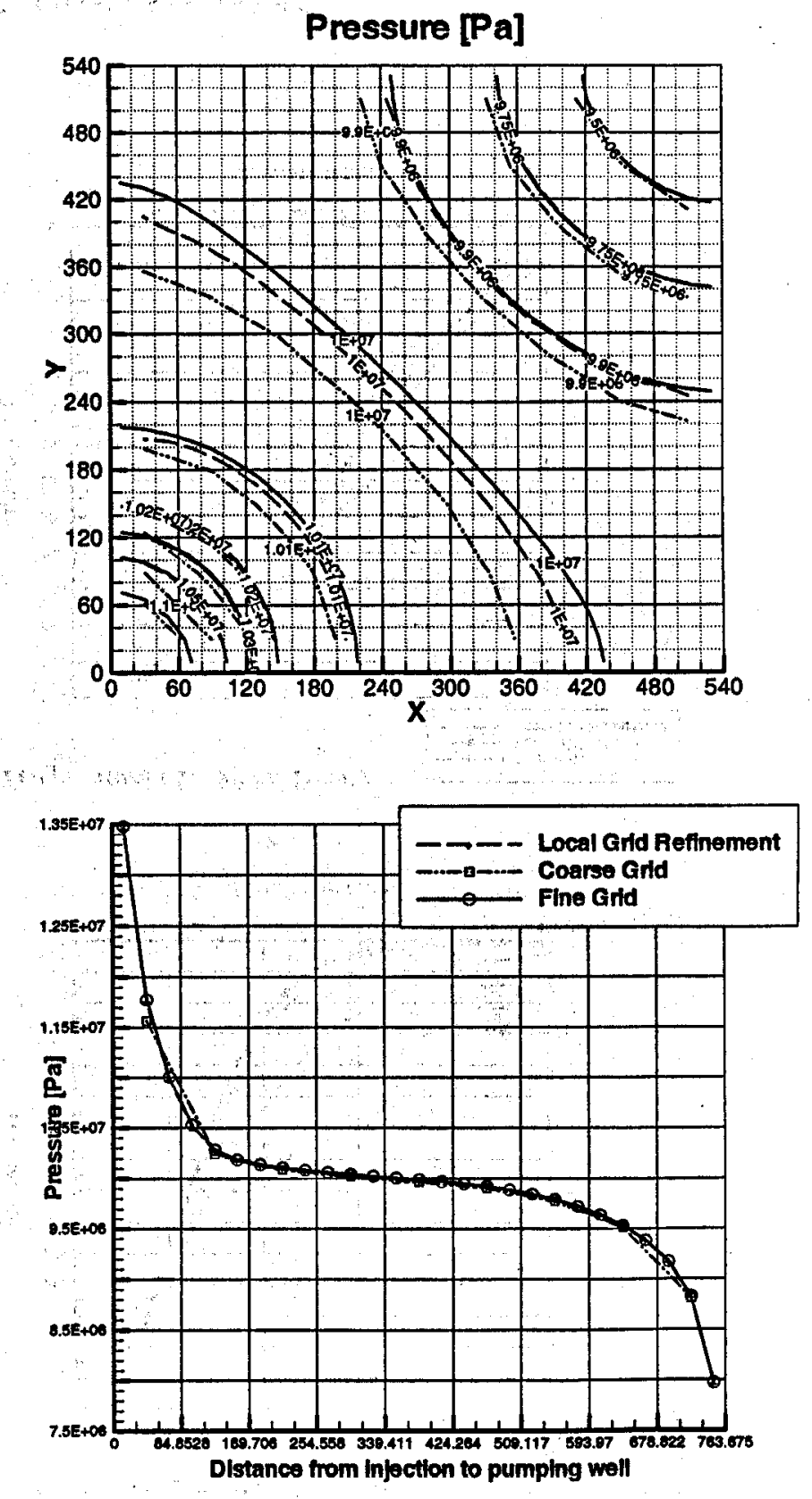

Saturation, temperature and pressure - Scenario 2 - Time $=2$ years 


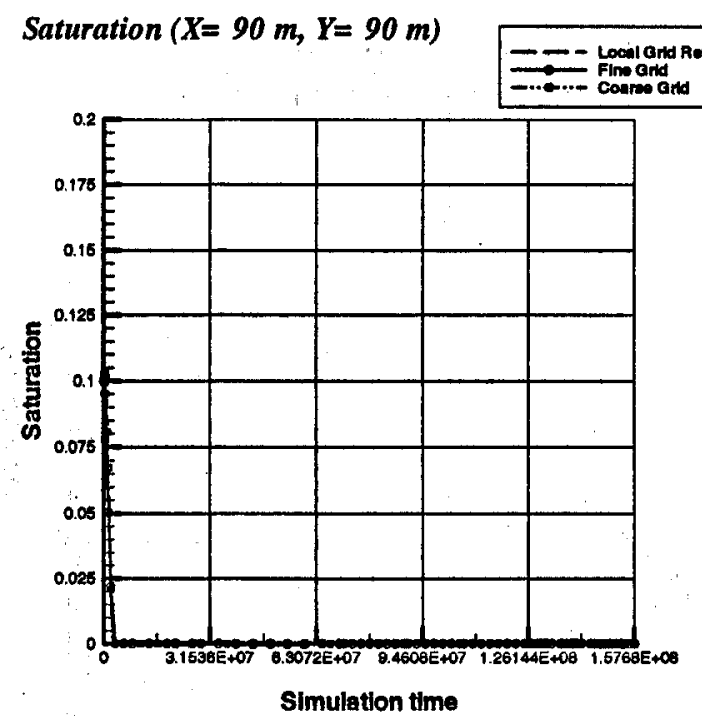

$\stackrel{\omega}{\not}$

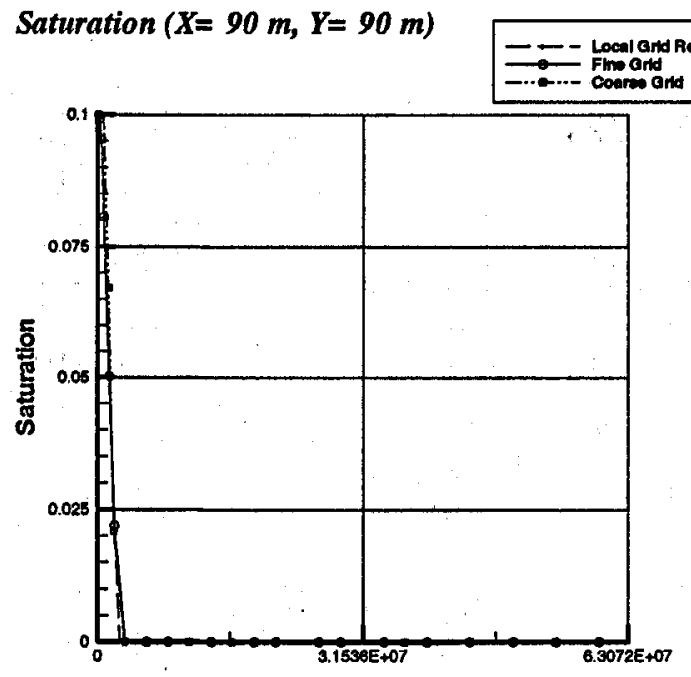

Simulation time
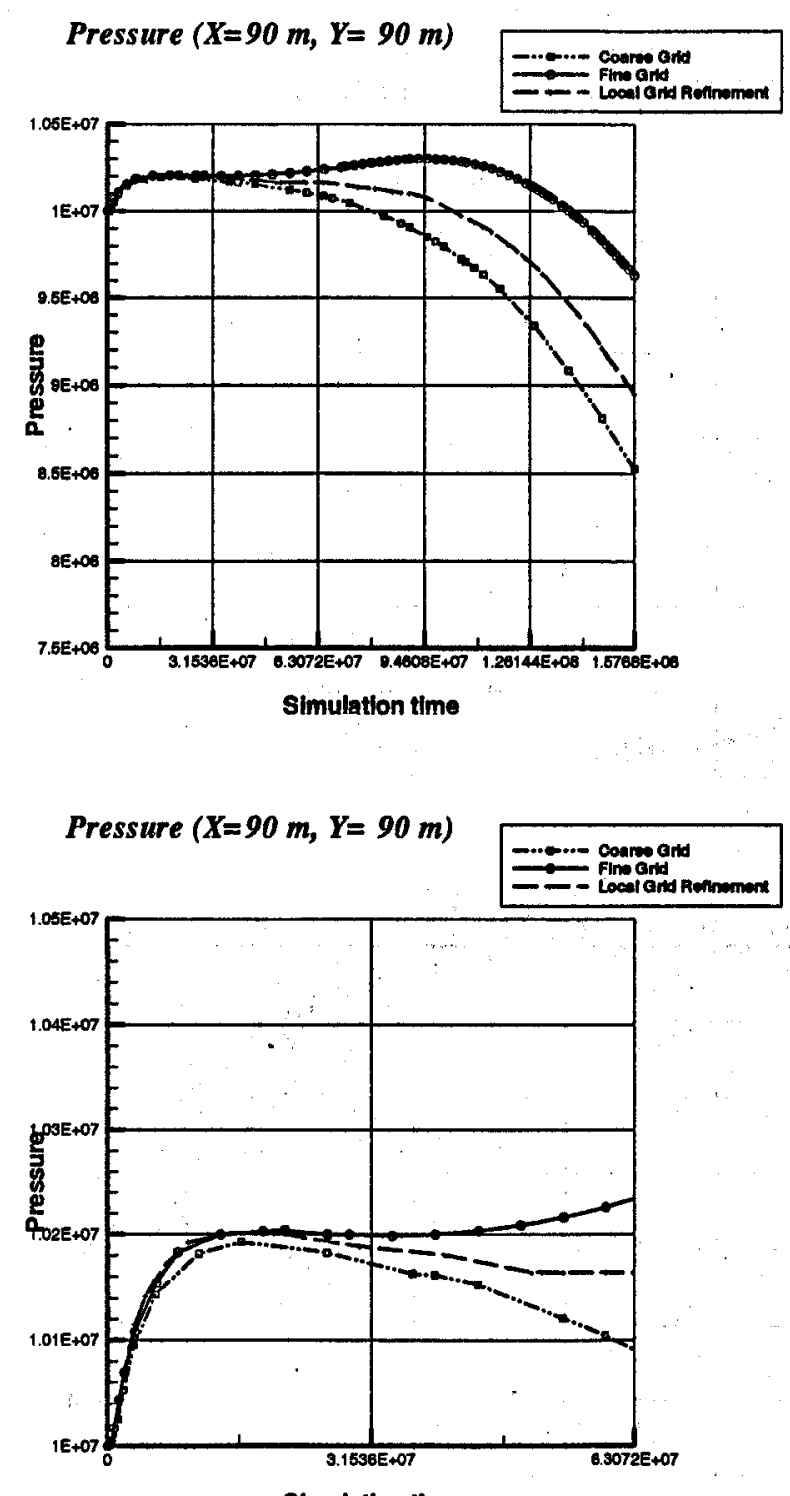

SImulation time

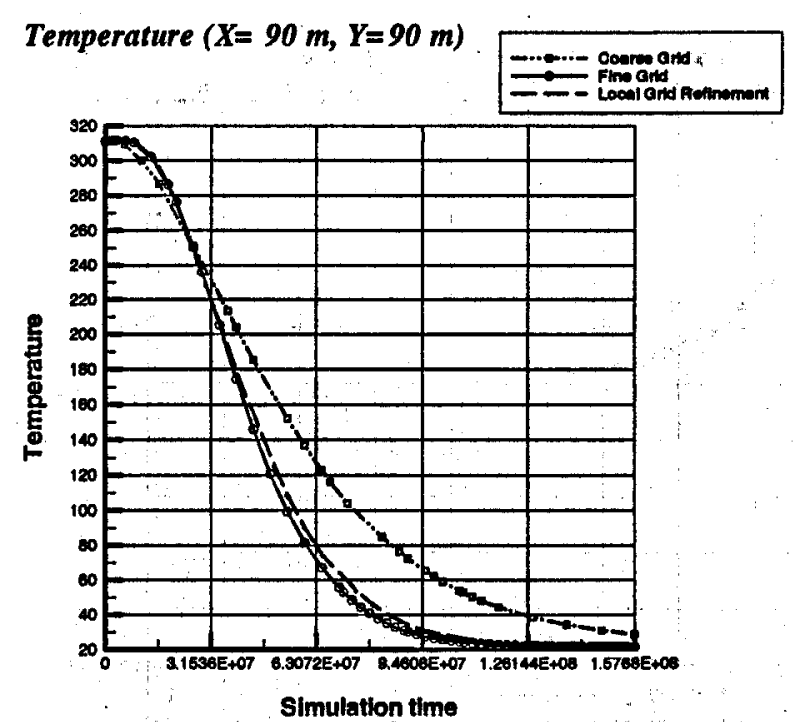

Temperature $(X=90 \mathrm{~m}, \mathrm{Y}=90 \mathrm{~m}) \quad \ldots$

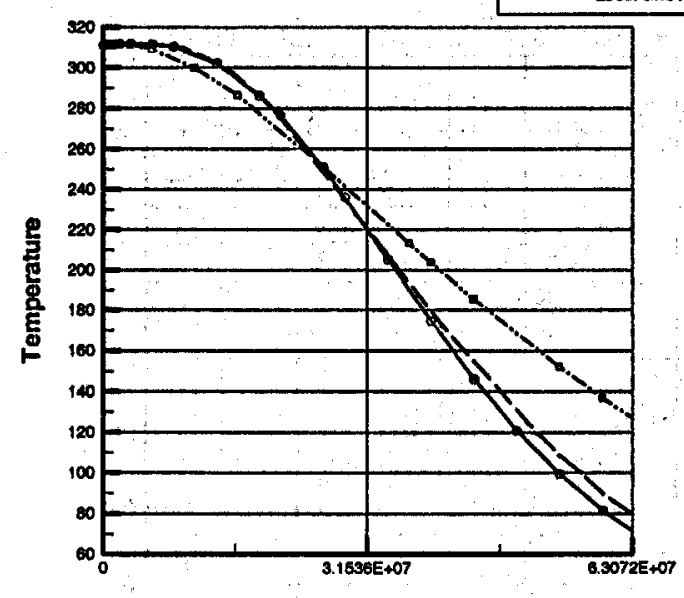

Simulation time

Figure 19

Saturation, pressure and temperature at a point near the injection well $(X=90 \mathrm{~m}, \mathrm{Y}=90 \mathrm{~m})$ - Scenario \# 2 
Saturation $(X=270 \mathrm{~m}, Y=270 \mathrm{~m})$

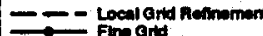 $=$ Fon and}

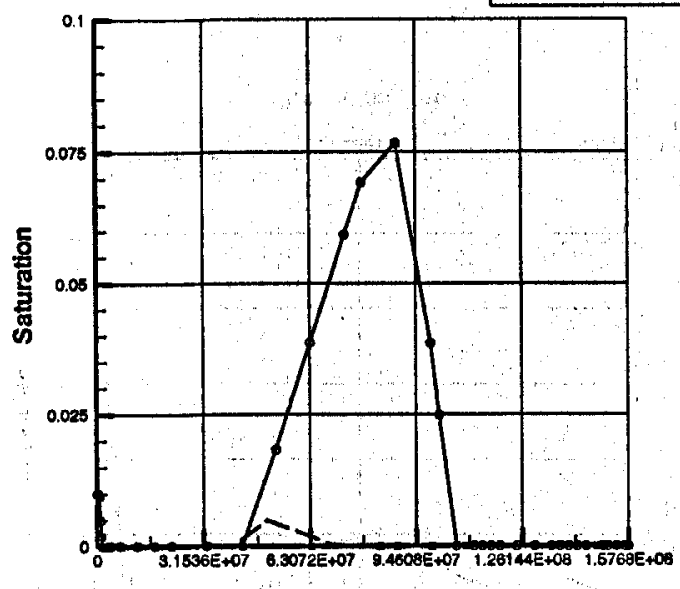

Simulation time

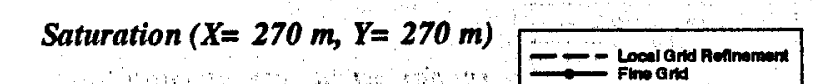

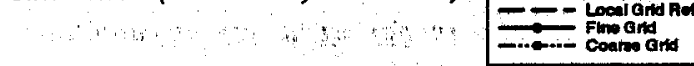

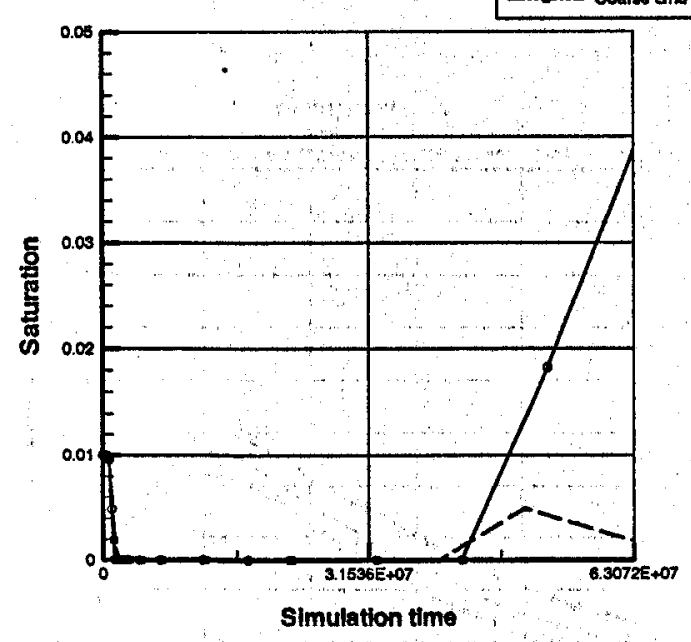

Pressure $(X=270 \mathrm{~m}, \mathrm{Y}=270 \mathrm{~m})$

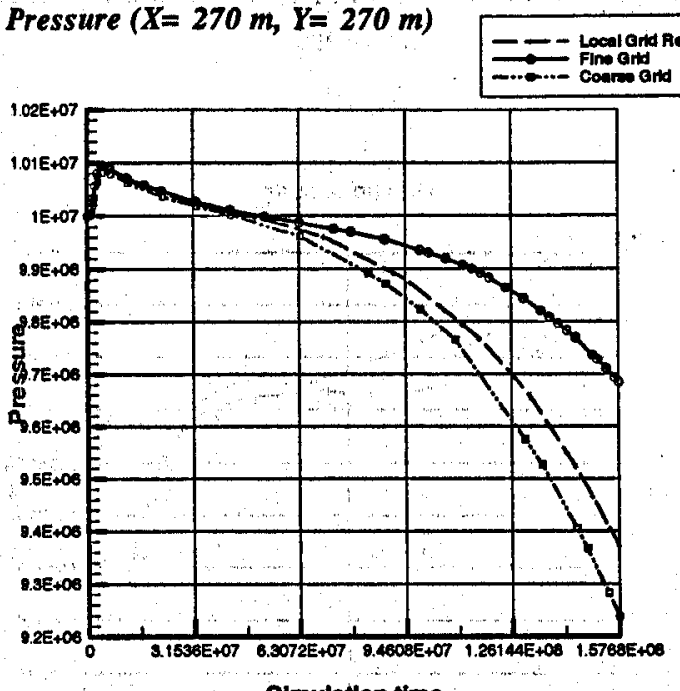

Simulation time
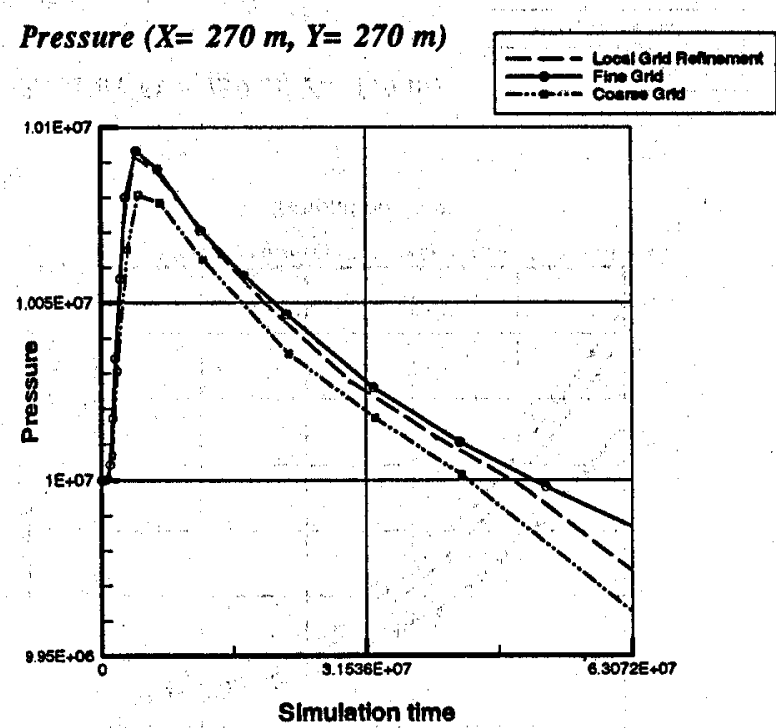

Temperature $(X=270 \mathrm{~m}, \dot{Y}=270 \mathrm{~m})$

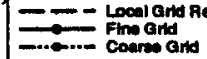

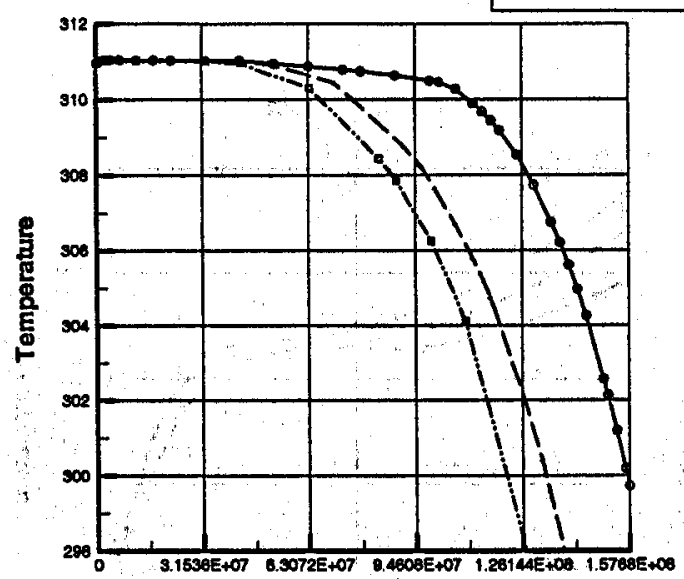

Simulation time
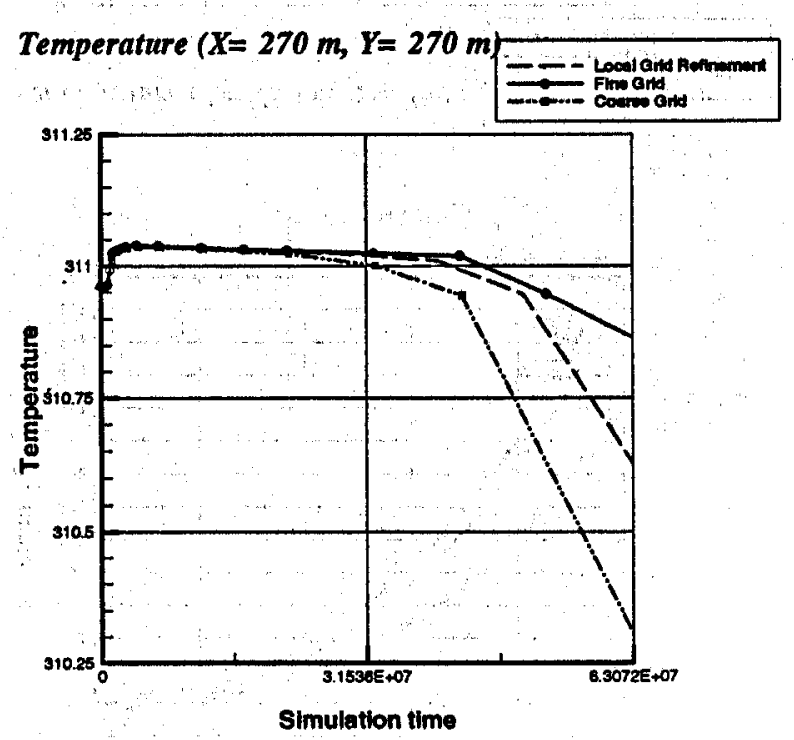

Figure 20

Saturation, pressure and temperature at the middle of the domain $(X=270 \mathrm{~m}, Y=270 \mathrm{~m})$ - Scenario 2 


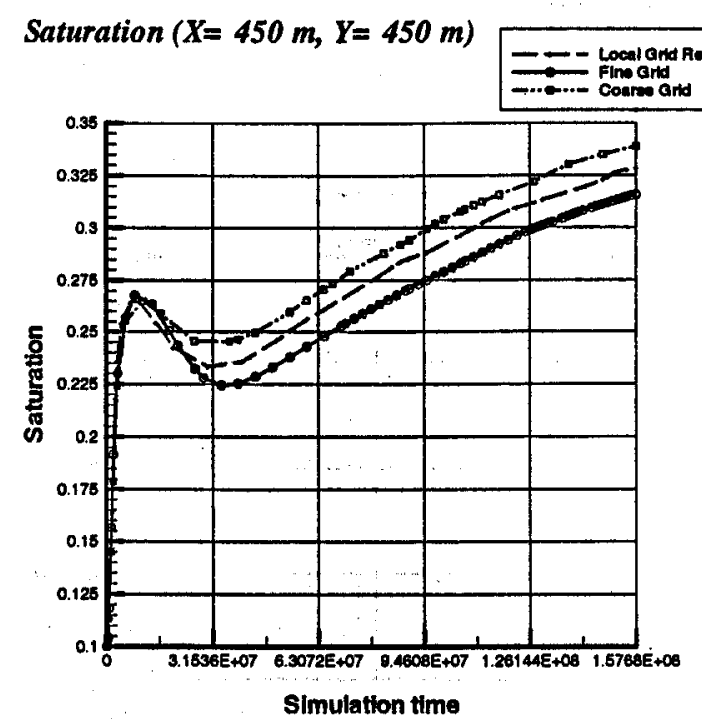

ì
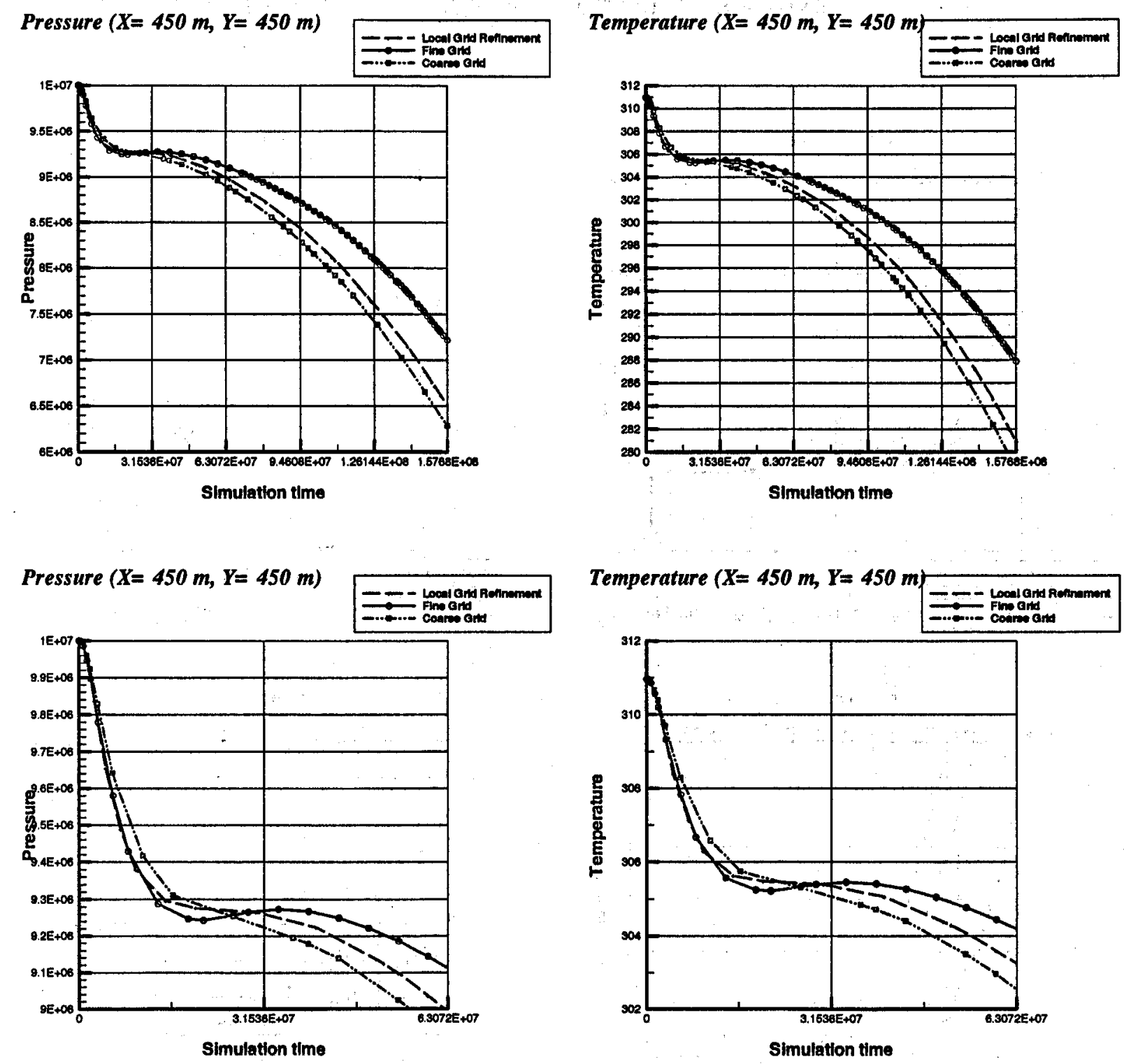

Figure 21

Saturation, pressure and temperature at a point near the production well $(X=450 \mathrm{~m}, Y=450 \mathrm{~m})$ - Scenario \# 2 


\section{Gas Saturation}

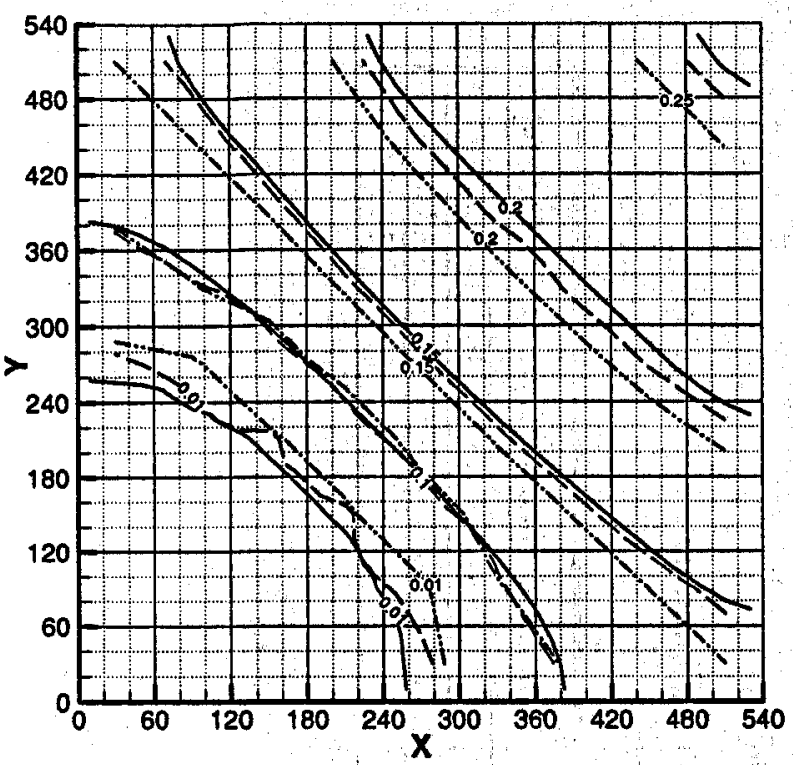

$\dot{w}$

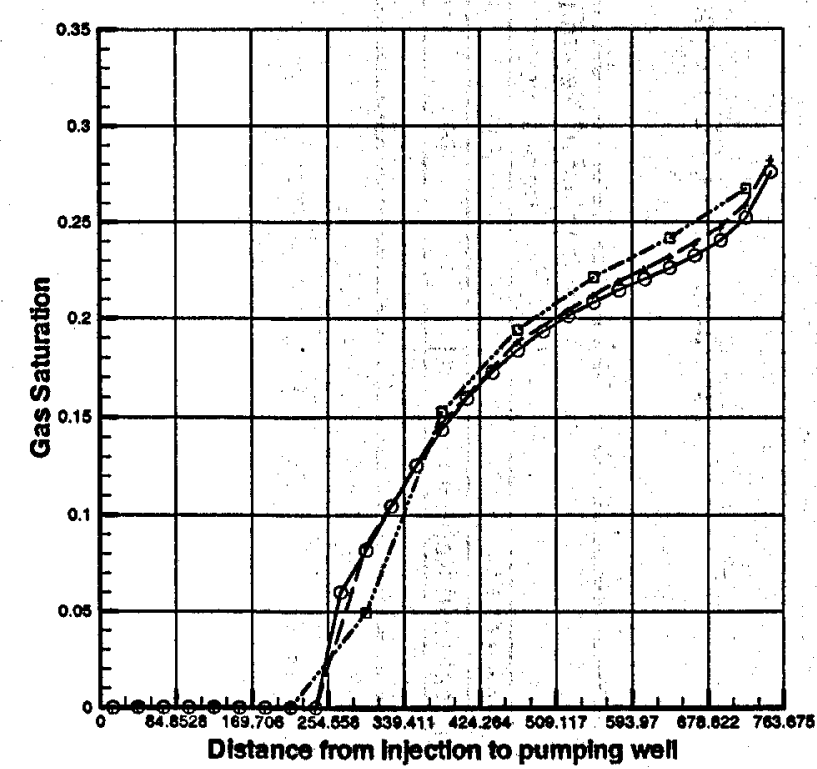

Temperature $\left.{ }^{\circ} \mathrm{C}\right]$


Figure 22
Pressure [Pa]
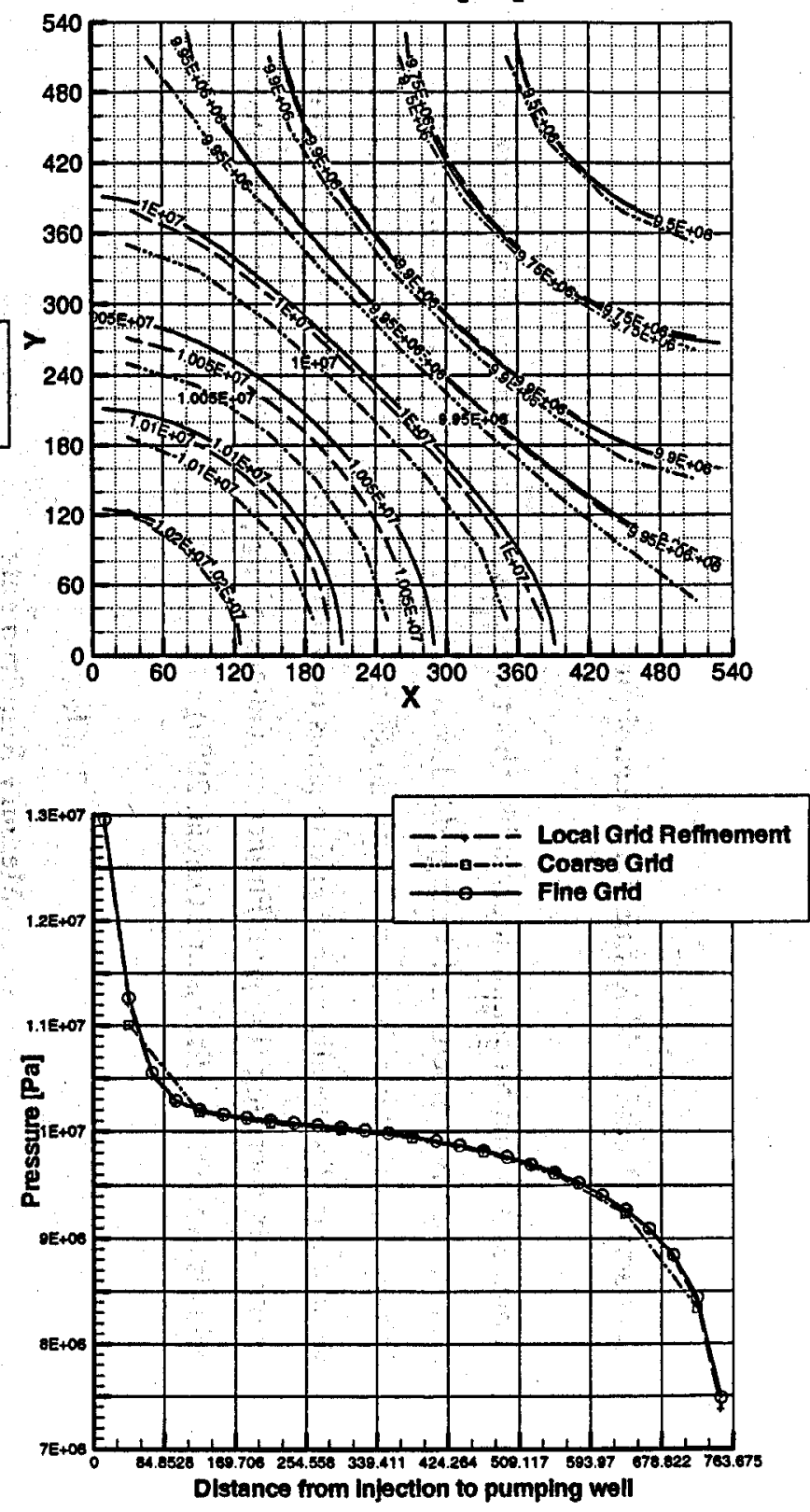

Saturation, temperature and pressure - Scenario \# 3 - Time = 1 year 


\subsection{Economy of Local Grid Refinement Techniques}

Tables 2, 3, 4 and 5 contain information about the Newton-Raphson iteration process for coarse, locally refined, and fine grids. Local grid refinement reduces the size of the Jacobian matrix by a factor of 3 with no sacrifice in accuracy relative to the fine grid simulations. The locally refined grid requires only about a quarter of the simulation time as the corresponding fine grid. Further evaluations of the performance of locally refined grids is available in (Pruess and García, 2000).

Table 2

Memory Requirements

\begin{tabular}{|l|c|c|c|c|}
\hline & $\begin{array}{c}\text { Number of } \\
\text { Elements }\end{array}$ & $\begin{array}{c}\text { Number of } \\
\text { Connections }\end{array}$ & $\begin{array}{c}\text { Jacobian Matrix } \\
\text { Dimension }\end{array}$ & $\begin{array}{c}\text { Number of non- } \\
\text { zero Jacobian } \\
\text { Terms }\end{array}$ \\
\hline Coarse Grid & 81 & 144 & 162 & 1476 \\
\hline Local Grid Refinement & 225 & 432 & 450 & 4516 \\
\hline Fine Grid & 729 & 1404 & 1458 & 14148 \\
\hline
\end{tabular}

Table 3

Scenario \# 1 - CPU Requirements (Simulation Time 5 years)

\begin{tabular}{|l|c|c|c|}
\hline & $\begin{array}{c}\text { Number of Newton } \\
\text { Iterations }\end{array}$ & Number of Time Steps & CPU Time [s] \\
\hline Coarse Grid & 88 & 21 & 1.700 \\
\hline Local Grid Refinement & 156 & 30 & 9.720 \\
\hline Fine Grid & 145 & 29 & 43.770 \\
\hline
\end{tabular}

Table 4

Scenario \# 2 - CPU Requirements (Simulation Time 2 years)

\begin{tabular}{|l|c|c|c|}
\hline & $\begin{array}{c}\text { Number of Newton } \\
\text { Iterations }\end{array}$ & Number of Time Steps & CPU Time [s] \\
\hline Coarse Grid & 64 & 16 & 1.260 \\
\hline Local Grid Refinement & 88 & 20 & 5.380 \\
\hline Fine Grid & 107 & 22 & 33.510 \\
\hline
\end{tabular}


Table 5

Scenario \# 3 - CPU Requirements (Simulation Time 1 year)

\begin{tabular}{|l|c|c|c|}
\hline & $\begin{array}{c}\text { Number of Newton } \\
\text { Iterations }\end{array}$ & Number of Time Steps & CPU Time [s] \\
\hline Coarse Grid & 54 & 14 & 1.150 \\
\hline Local Grid Refinement & 85 & 19 & 5.550 \\
\hline Fine Grid & 85 & 18 & 26.530 \\
\hline
\end{tabular}

\subsection{CONCLUSIONS}

We have presented a systematic approach to local grid refinement for the TOUGH2 simulator, which involves the use of special "interpolation nodes" at the boundary between refined and unrefined regions. The method was implemented into TOUGH2 for 2-D Cartesian grids. Application to geothermal production and injection problems has demonstrated much improved numerical accuracy in comparison to unrefined grids, at considerable savings in computer memory and execution time compared to globally refined grids. Future work should consider local refinement for fully 3-D grids. 


\section{Acknowledgement}

For a review of the manuscript and suggestions of improvements, thanks are due to Curt Oldenburg and Tianfu Xu. This work was supported by the Assistant Secretary for Energy Efficiency and Renewable Energy Office of Geothermal and Wind Technologies, of the U.S. Department of Energy under Contract No. DE-AC03-76SF00098. 


\section{References}

Aziz, K., Settari, A., 1979, Petroleum Reservoir Engineering. Elsevier, London and New York.

Chen, W.L., Lien, F.S., Leschziner, M.A., 1997 Local mesh refinement within a multi-block structured-grid scheme for general flows, Computer methods in Applied Mechanics and Engineering, 144 (1997) 327-369.

Hermitte, T., Guerillot, D.R., 1993, A more accurate numerical scheme for locally refined meshes in heterogenous reservoirs. SPE 25261, $12^{\text {th }}$ SPE Symposium on reservoir Simulation. New Orleans, Feb. 28-March 3, 1993, 321-331.

Lazarov, R. D., Mishev, I D., Vassilevski, P. S., 1994, Finite volume methods with local refinement for convection-diffusion problems. Computing (53), (1994), 33-57.

Moridis, G.J., Pruess, K., 1998, T2SOLV: An enhanced package of solvers for the TOUGH2 family of reservoir simulation codes, Geothermics, Vol. 27, No. 4, (1998), 415444.

Narasimham, T.N., Witherspoon, P.A., 1976, An integrated finite difference method for analyzing fluid flow in porous media, Water Resources Research, Vol. 12, No. 1, 57-60, 1976.

Peaceman, D.W., 1977. Fundamentals of Numerical Reservoir Simulation, Elsevier, Amsterdam.

Pruess, K., 1987. TOUGH User's Guide, Lawrence Berkeley Laboratory report LBL-20700.

Pruess, K, 1991a. Grid orientation and capillary pressure effects in the simulation of water injection into depleted vapor zones, Geothermics, Vol.20, No. 5/6, 257-277, 1991.

Pruess, K, 1991b. TOUGH2 - A General Purpose Numerical Simulator for Multiphase Fluid and Heat Flow, Lawrence Berkeley Laboratory report LBL-29400.

Pruess, K., Oldenburg, C., Moridis, G., 1999. TOUGH2 User's Guide, Version 2.0, Lawrence Berkeley National Laboratory report LBNL-43134.

Pruess, K., García, J.E., 2000. A systematic approach to local grid refinement in geothermal reservoir simulation, Proceedings of the World Geothermal Congress 2000, May 28 - June 10, 2000 (in press.) 


\section{APPENDIX A: DEFINITIONS}



Composite connection: a connection is said to be composite if it involves a special block and a refined block.

Composite grid: a grid is said to be composite if it is composed of some refined blocks.

Interpolation Node: A fictitious node created to compute fluxes between special blocks. (i.e. across regions with different refinement.)

Reference Element: Neighboring grid block that will be used for interpolation.

Refined block: a block is said to be refined if it is subdivided into $\mathrm{n}^{2}$ equal volume blocks, where $\mathrm{n}$ is the Coefficient of refinement.

Regular grid: a grid is said to be regular if there is no refined block.

Special block: a block is said to be special if at least one neighboring block has a node not aligned with the original one. 


\section{APPENDIX B: TOUGH2 INPUT FORMATS FOR GRID GENERATION AND GEOMETRIC DESCRIPTION}

\section{Geometric Description}

ELEME introduces element (grid block) information

\section{Record ELEME.1}

Format (A3, I2, 2I5, A3, A2, 6e10.4)

EL, NE, NSEQ, NADD, MA1, MA2, VOLX, AHTX, PMX, X, Y, Z

EL, NE 5-character code name of an element. The first three characters are arbitrary, the last two characters must be numbers.

NSEQ number of additional elements having the same volume and belonging to the same reservoir domain.

NADD increment between the code numbers of two successive elements. (Note: the maximum permissible code number NE+NSEQ*NADD is $\leq 99$.).

MA1, MA2 a five character material identifier corresponding to one of the reservoir domains as specified in block ROCKS. If the first three characters are blanks and the last two characters are numbers then they. indicate the sequence number of the domain as entered in ROCKS. If both MA1 and MA2 are left blank the element is by default assigned to the first domain in block ROCKS.

VOLX element volume $\left(\mathrm{m}^{3}\right)$.

AHTX interface area $\left(\mathrm{m}^{2}\right)$ for heat exchange with semi-infinite confining beds. Internal MESH generation via MESHMAKER will automatically assign AHTX.

PMX permeability modifier (optional, active only when a domain 'SEED' has been specified in the ROCKS block). Will be used as multiplicative factor of the permeability parameters from block ROCKS. Simultaneously, strength of capillary pressure will be scaled as $1 / \mathrm{SQRT}(\mathrm{PMX}) . \mathrm{PMX}=0$ is permitted and will result in an impermeable block.

$\mathrm{X}, \mathrm{Y}, \mathrm{Z}$ Cartesian coordinates of grid block centers. These may be included in the ELEME data to make subsequent plotting of results more convenient. Generally speaking, the coordinate data are not used in TOUGH2; the single exception is for optional initialization of gravity-capillary equilibrium with EOS9.

Record ELEME. 2 A blank record closes the ELEME data block. 
CONNE introduces information for the connections (interfaces) between elements.

Record CONNE.1

Format(A3, I2, A3, I2, 4I5, 5E10.4, 2X,I5,2I3)

EL1, NE1, EL2, NE2, NSEQ, NAD1, NAD2, ISOT, D1, D2, AREAX, BETAX, SIGX, PHAN, ICO, CRE

EL1, EL2 code name of the first element

EL2, NE2 code name of the second element

NSEQ number of additional connections in the sequence

NAD1 increment of the code number of the first element between two successive connections

NAD2 increment of the code number of the second element between two successive connections.

ISOT set equal to 1,2 , or 3 ; specifies absolute permeability to be PER(ISOT) for the materials in elements (EL1, NE1) and (EL2, NE2), where PER is read in block ROCKS. This allows assignment of different permeabilities, e.g., in the horizontal and vertical direction.

D1 J distance (m) from center of first and second element, respectively,

D2 to their common interface.

AREAX interface area $\left(\mathrm{m}^{2}\right)$. Note: for locally refined grids, set the area to a negative value to identify composite connections and the presence of an interpolation node)

BETAX cosine of the angle between the gravitational acceleration vector and the line between the two elements. GF*BETAX $>0(<0)$ corresponds to first element being above (below) the second element.

SIGX "radiant emittence" factor for radiative heat transfer, which for a perfectly "black" body is equal to 1 .

PHAN active if AREAX $<0$. Reference element for composite connections.

ICO active if AREAX $<0$. Location index of the interpolation node.

CRE active if AREAX $<0$. Coefficient of refinement required to calculate appropriate interpolation factors for interpolation node.

Repeat record CONNE.1 for the number of connections desired.

Record CONNE.2 A blank record closes the CONNE data block.

For additional information see Figure 23 


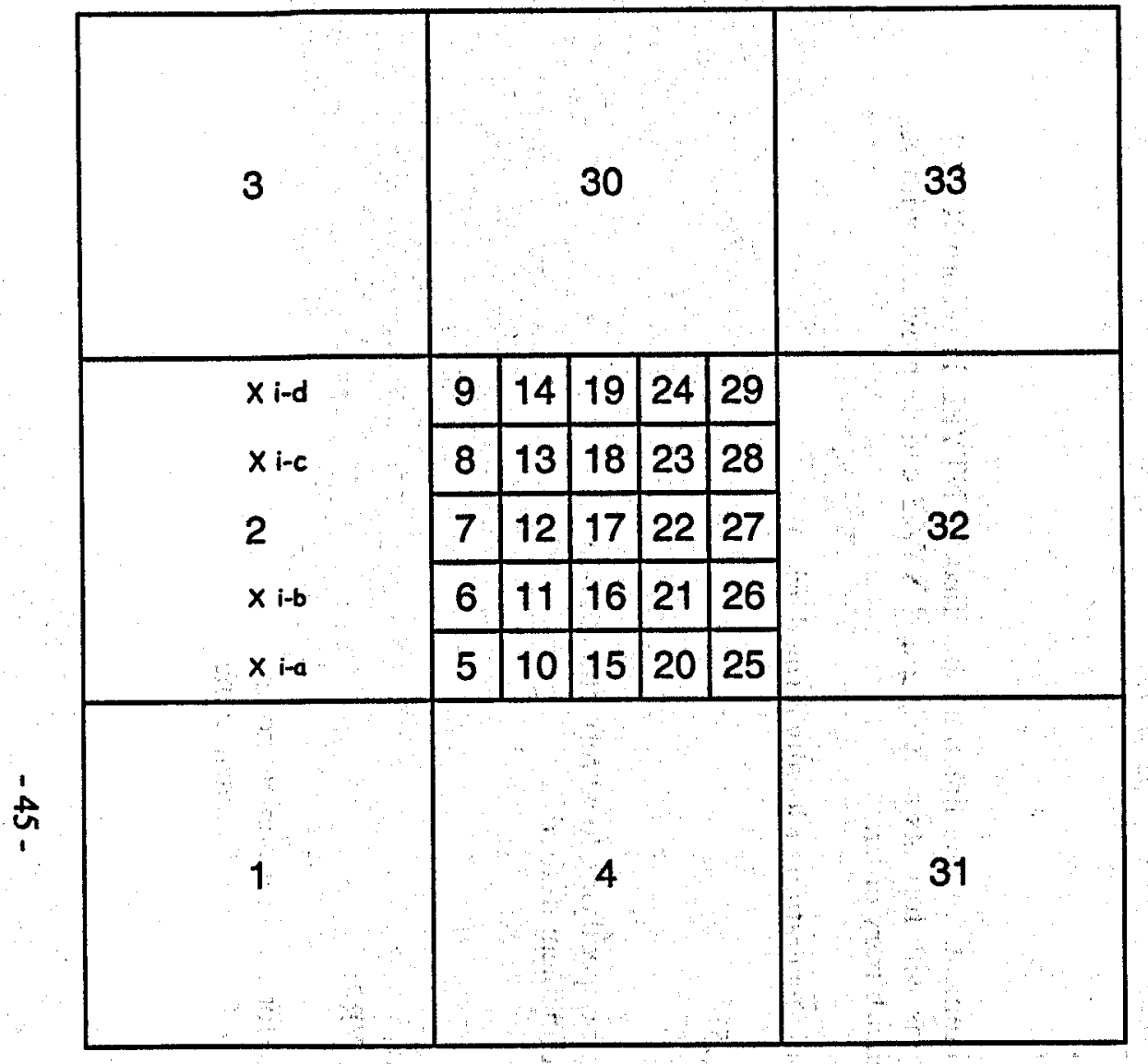

Figure 24

Illustration of MESH data for locally refined grid
$10.54008+050.72008+04$ $10.54008+050.72008+04$

$10.54008+050.72008+04$

$10.54002+050.72002+0$

$10.21608+040.2880 \mathrm{~B}+03$

$10.21608+040.28808+03$

$10.21608+040.28802+03$

$10.21608+010.28808+03$

$10.21608+040.28808+03$

$10.21608+040.28808+03$

$10.21608+010.28808+03$

$10.21608+040.28808+03$

$10.21608+040.28808+03$

$10.21608+040.28808+03$

$10.21608+040.2880 B+03$

$10.21608+040.26808+03$

$10.21608+040.28808+03$

$10.54008+050.72008+04$
$.30008+020.30008+02-.75008+01$ $0.30008+020.90008+02-.75008+01$ $0.30008+020.15008+03-.75008+01$ $0.9000 z+020.30008+02-.7500 z+01$ $0.6600 \mathrm{E}+020.66008+02-.7500 \mathrm{E}+02$ $0.6600 \mathrm{E}+020.7800 \mathrm{E}+02-.7500 \mathrm{E}+01$ $0.66008+020.90008+02-.75008+01$ $0.66008+020.10202+03-.75002+0$ $0.66008+020.11408+03-.75008+01$

$0.78008+020.66008+02-.75008+01$ $0.78008+020.78008+02-.75002+01$ $0.78008+020.90008+02-.75008+01$ $0.78008+020.10208+03-.75008+0$ $0.7800 \mathrm{E}+020.11408+03-.7500 \mathrm{E}+01$ $0.90008+020,66003+02-.75008+01$ $0.90008+020.78008+02-.75008+02$ $0.9000 \mathrm{E}+020.9000 \mathrm{~B}+02-.7500 \mathrm{E}+0 \mathrm{Z}$

$$
\therefore
$$

$0.1500 \mathrm{~B}+030.1500 \mathrm{~B}+03-.7500 \mathrm{~B}+01$

Element 2 has 5 composite connections. To calculate fluxes across the composite connections four interpolations nodes are required (i-a, i-b, i-c, i-d). Reference element for $i-a$ and $i-b$ is 1 . Reference element for $i-c$ and $i-d$ is 3 .

for $c$ and $\mathrm{i} d \mathrm{is}$. Location Index for $i-b$ and $i-c$ is $L I=1$ Location Index for $i-a$ and $i-d$ is $L I=2$ Coefficient of Refinement is 5 (CR=5) Coefficients of interpolation for $i-a$
$10.30008+020.30008+020.9000 z+03$

$20.3000 \mathrm{E}+020.30008+020.90008+030.0000 \mathrm{E}+00$

$10.3000 z+020.60008+01-.18008+03$

10.30008+020.60008 $+01-.18008+03$

$10.30008+020.60008+01-.18008+03$

$10.3000 \mathrm{z}+020.6000 \mathrm{~B}+01-.1800 \mathrm{~B}+03$

$10.30008+020.6000 \mathrm{~B}+01-.1800 \mathrm{~B}+03$

$20.30008+020.3000 E+020.90008+030.00008+00$

$10.30008+020,30008+020.90008+03$

$10.30008+020.3000 B+020.9000 B+03$

$20.30008+020.60008+01-.18008+030.00008+00$

$20.30008+020,60008+01-.18008+030.00008+00$

$20.3000 \mathrm{~B}+020.6000 \mathrm{R}+01-.1800 \mathrm{~B}+030.0000 \mathrm{~B}+00$

$20.3000 \mathrm{~B}+020.6000 \mathrm{~B}+01-.1800 \mathrm{~B}+030.0000 \mathrm{~B}+00$

$20.3000 \mathrm{~B}+020.6000 \mathrm{~B}+01-1800 \mathrm{~B}+030.00008+00$

$10.60008+010.60008+010.18008+63$

$20.3000 \mathrm{~B}+020,3000 \mathrm{~B}+020,9000 \mathrm{R}+030.0000 \mathrm{~B}+00$ and $i-d$ is $2 / 5$ (LI/CR) and 1-2/5

Coefficients of interpolation for $i-b$ and $i-c$ is $1 / 5$ (LI/CR) and $1-1 / 5$
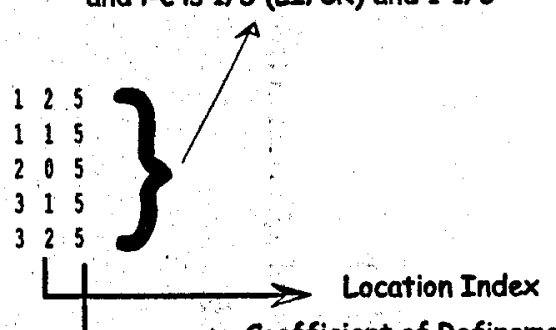

125

115

105

3115

$\longrightarrow$ Reference Element

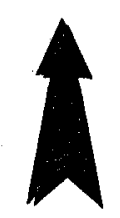

Additional Information in Connnection Data 


\section{Mesh Generation (Cartesian Grids)}

XYZ invokes generation of a Cartesian (rectilinear) mesh

\section{$\underline{\text { Record XYZ.1 }}$}

Format(E10.4)

DEG

DEG

angle (in degrees) between the $Y$-axis and the horizontal. If gravitational accelaration (parameter GF in record PARAM.2) is specified positive, $-90^{\circ}<$ DEG $<90^{\circ}$ corresponds to grid layers going from top down. Grids can be specified from bottom layer up by setting GF or BETA negative. Default $(\mathrm{DEG}=0)$ corresponds to horizontal $\mathrm{Y}$ - and vertical $\mathrm{Z}$-axis. $\mathrm{X}$-axis is always horizontal.

$\underline{\text { Record XYZ.2 }}$

Format (A2, 3X, I5, E10.4)

NTYPE, NO, DEL

NTYPE

set equal to $\mathrm{NX}, \mathrm{NY}$ or $\mathrm{NZ}$ for specifying grid increments in $\mathrm{X}, \mathrm{Y}$, or $\mathrm{Z}$ direction.

NO

number of grid increments desired

DEL

constant grid increment for NO grid blocks, if set to a non zero valule.

Record XYZ.3 (optional, $\mathrm{DEL}=0$. or blank only)

Format (8E10.4)

DEL(I), I=1, NO

DEL(I) a set of grid increments in the direction specified by NTYPE in record XYZ.2. Additional records with formats as XYZ.2 and XYZ.3 can be provided, with X, $\mathrm{Y}$, and Z-data in arbitrary order.

$\underline{\text { Record XYZ.4 (optional, generation of locally refined grids) }}$

Format(A2,3X,I5)

NTYPE, CRLRZ

NTYPE set equal to LR for specifying local refinement zone

CRLRZ coefficient of refinement desired

$\underline{\text { Record XYZ.5 }}$

Format(4E10.4)

$\mathrm{XR} 1, \mathrm{XR} 2, \mathrm{YR} 1, \mathrm{YR} 2$

left $\mathrm{x}$ coordinate for local refinement zone.

XR2

right $x$ coordinate for local refinement zone. 
YR1

lower y coordinate for local refinement zone.

YR2

upper y coordinate for local refinement zone.

Repeat record XYZ.4 for additional refinement zones desired.

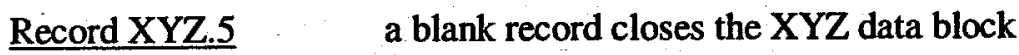

